\title{
Dynamic Catalysis Fundamentals: II. Consequences of scaling relationships on mechanisms and kinetics
}

Brandon L. Foley,"* and Neil K. Razdan ${ }^{\mathrm{b}}$

${ }^{\mathrm{a}}$ Lawrence Livermore National Laboratory, 7000 East Avenue, Livermore, California 94550

${ }^{b}$ Department of Chemical Engineering and Materials Science, University of Minnesota, Twin Cities, Minneapolis, MN 55455

*Corresponding Author: E-mail: brfoley12@gmail.com 


\begin{abstract}
Dynamic catalysis proffers a new strategy for leveraging linear free energy (LFE) relationships in catalysis to increase reaction rate, conversion, and selectivity by high-frequency, forced kinetic oscillations. This work explicates two dynamic catalysis mechanisms - "resonance" and quasi-static, characterized by finite frequency bands and high frequency limits, respectively — and details the necessary LFE parameters necessary for each dynamic catalysis phenomena to arise. Detailed analytical and numerical analyses reveal that under quasi-static mechanisms, Sabatier limits on reaction rates and thermodynamic limits on conversion can be completely subverted with sufficiently large kinetic oscillation amplitudes. In resonance mechanisms, reaction rates and conversion are still limited by Sabatier volcanos and thermodynamic equilibrium constants, respectively; however, these imposed limitations are those for a subset of elementary steps, rather than for the entire overall reaction. An investigation of dynamic catalysis for reaction schemes with multiple products reveals that quasi-static dynamic catalysis can drive selectivities of any intermediate or product to $100 \%$, provided the appropriate LFE relationships.
\end{abstract}

\title{
1. Introduction
}

The advent of computational catalysis and electronic structure calculation methods has facilitated the discovery of quantitative reactivity trends amongst transition-metal catalysts which affirm decades-old postulated linear scaling relationships between energies of surface-bound intermediates and kineticallyrelevant transition states [1-3]. These linear free energy (LFE) relationships simplify the microkinetic description of catalytic reaction networks down to a small number of pertinent LFE scaling parameters and, in doing so, quantitatively validate the Sabatier principle: that turnover rate is maximized at moderate substrate binding energies which balance kinetic driving forces for (i) reactant adsorption/reaction and (ii) product desorption $[4,5]$. This simple, intuitive principle has proven virtuous in rationalizing observed reactivity trends across a diverse range of catalytic chemistries and transition metal surfaces $[4,6-10]$. The ubiquity of the Sabatier prescription, affirmed experimentally and quantitatively reconciled by $a b$ initio quantum mechanical calculation methods, reinforces the need to develop new strategies to "break" linear 
scaling and overcome conventional limits to catalytic turnover rate [11-14]. Recently, dynamic catalysisthe forced oscillation of elementary step rate constants - has emerged as a promising method to both (i) accelerate heterogeneously-catalyzed reactions beyond the Sabatier limit and (ii) drive chemical conversion to supra-equilibrium values by exploit of thermodynamic work introduced by, for example, a fluctuating electric potential $[14,15]$. Theoretical [16-21] and experimental [22-26] studies have substantiated the competence of dynamic strategies to enhance catalytic turnover rates by orders of magnitude compared to static systems, but the fundamental precepts and catalyst-design considerations which dictate the efficacy of dynamic strategies remains elusive. To this end, we build upon the mathematical framework we recently developed to derive quantitative guiding principles for dynamic catalysis which, analogous to the Sabatier principle for static systems, prescribe the kinetic consequences of LFE scaling for dynamic catalytic performance and behavior [16].

In brief, we facilitate analytical derivation and numerical discovery of rates and coverages at the "dynamic steady state", or limit cycle, by judicious application of periodic and continuity boundary conditions to the governing set of differential equations describing the frequency response of each surface intermediate. This solution technique (i) disencumbers numerical approaches of the requirement to calculate computationally-onerous transients preceding the limit cycle [18] and (ii) enables the derivation of closedform relationships between LFE scaling parameters and enhancement to rate, selectivity, and/or conversion. From inspection of these simple, interpretable analytical relations, we identify two limiting cases, the quasistatic and resonance conditions, which manifest as distinct mechanisms of dynamic catalysis with disparate characteristic frequency response, kinetic prerequisites, and opportunities for optimization [16].

The quasi-static mechanism of dynamic catalysis is defined by rate enhancement at the high frequency limit, where forced kinetic oscillations occur much more rapidly than elementary step interconversion of surface species. Consequently, surface coverages are effectively time-invariant, or quasistatic. The time-invariance of adspecies concentrations during quasi-static dynamic catalysis confers significant simplifications to analytical description which we exploit to derive, in closed-form, the exact 
consequences of LFE scaling relations on rate, selectivity, and the capacity to achieve supra-equilibrium conversion.

The resonance mechanism of dynamic catalysis, observed in precedent and contemporary theoretical/computational studies [16-18,20,21,27], refers to dynamic systems that exhibit a finite band of apparent "resonant" frequencies within which turnover rate is maximized. We re-interpret catalytic resonance phenomena in the context of the described mathematical framework to (i) develop an understanding of the precise mechanism by which kinetic systems exhibit resonance behavior, (ii) demonstrate that resonance follows directly from and is a manifestation of LFE relations underpinning the Sabatier principle, (iii) derive the kinetic and thermodynamic requirements for resonance in terms of linear scaling parameters, and (iv) establish a method for deriving rate expressions for resonance catalysis which, in some cases, are identical to Langmuir-Hinshelwood descriptions of conventional static catalytic reactions.

We synthesize learnings from description of the quasi-static and resonance conditions to identify, for each set of LFE parameters, the dynamic strategy (quasi-static or resonance) which most effectively accelerates a generic three-step catalytic reaction $\left(\mathrm{A}+{ }^{*} \rightleftharpoons \mathrm{A}^{*} \rightleftharpoons \mathrm{B}^{*} \rightleftharpoons \mathrm{B}+*\right)$. In doing so, we generate a map of systematically-classified dynamic catalysts, which, despite all being described by the Sabatier principle at static conditions, exhibit distinct dynamic behavior. We contend that this classification of dynamic catalysts provides a straight-forward guide for future efforts which seek to understand and/or engineer catalytic systems and conditions most amenable to dynamic forcing strategies.

\section{Methods}

This work used Matlab version R2020a to run numerical calculations to study dynamic catalysis rate and conversion. This section details the methods employed in these codes. Example codes are available in Section S1 of the Supporting Information and are downloadable from https://github.com/foley352/DC-2. 


\subsection{Numerical methods for calculating dynamic catalysis rate at a specified condition}

Numerical methods employed herein utilize insights from our previous work [16], which explicates fast ( $<1 \mathrm{~ms}$ per condition) linear algebra-based methods for calculating rates and coverages at the "dynamic steady state", or limit cycle. Briefly, the governing ordinary differential equations (ODEs) describing any linear dynamic catalytic system are recast in terms of a single first-order matrix differential equation (eq. (1)):

$$
\frac{\mathrm{d} \boldsymbol{\theta}}{\mathrm{d} t}=\boldsymbol{A}(t) \boldsymbol{\theta}+\boldsymbol{b}(t)
$$

where $\boldsymbol{A}$ is a time-dependent coefficient matrix of rate constants and chemical activities of fluid-phase species, $\boldsymbol{\theta}$ is a vector of the fractional coverages of the linearly-independent surface species (one species is eliminated by virtue of $\sum \theta_{j^{*}}=1$ ), and $\boldsymbol{b}$ is a time-dependent vector of rate constants and fluid-phase species activities. The time-dependence of $\boldsymbol{A}$ and $\boldsymbol{b}$ proscribes a general solution-a complication we resolve by considering discretizing the forced oscillation as a concatenation of infinitesimal square waves (see Scheme 1) where $\boldsymbol{A}$ and $\boldsymbol{b}$ are time-invariant except at the step discontinuities (i.e., transition between square waves). The eigenvalues of the discretized matrices $\boldsymbol{A}$ quantify the characteristic timescales of chemical transformations and thus determine frequencies of forced oscillation required to enhance reaction rate and conversion. Equation (1), once discretized, is solved over the period, or wavelength $(\lambda)$, of the limit cycle by application of continuity conditions at each discretization step and periodic boundary conditions at $t$ and $t+\lambda$. With fractional coverages of all species in hand, the time-averaged rate is calculated by eq. (2):

$$
\langle r\rangle=\frac{\int_{t}^{t+\lambda} r \mathrm{~d} t^{\prime}}{\int_{t}^{t+\lambda} \mathrm{d} t^{\prime}}
$$


Scheme 1. An example of a square-wave oscillation between two kinetic states.

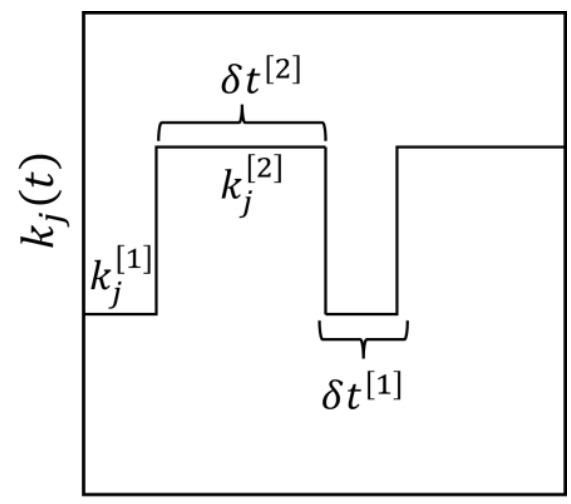

Time

\subsection{Numerical method for calculating the maximum dynamic catalysis rate}

The dynamic catalysis reaction rate is a function of the oscillation wave endpoints, frequency, and temporal asymmetry for a two-stepped square-wave oscillation. The optimal operating conditions to achieve the global maximum dynamic catalysis rate is found by sampling oscillation endpoints on the range of $-10<\Delta G_{2}^{\mathrm{o \neq}[j]} / R T<10$ and frequencies at three conditions $-f \rightarrow 0, f \rightarrow \infty$, and $f=\sqrt{\lambda_{1}^{[2]} \lambda_{2}^{[2]}}$, which is the center of the resonance regime as discussed in Section 3.2.3. The built-in Matlab function fminunc is then used to find the local maximum rate by varying the oscillation endpoints, frequency, and $\delta t^{[2]} / \delta t^{[1]}$. This method does not always find the global maximum, giving rise to a few artifacts in Figure 2 and Figure 4.

\section{Results and Discussion}

\subsection{Dynamic enhancement of conversion and rate for a two-step reaction sequence}

With the aim of developing a general understanding of catalyst properties necessary for achieving supra-Sabatier peak reaction rates and supra-equilibrium conversions, we first derive analytical solutions that describe the simplest dynamic catalysis system with reaction kinetics described by linear free energy 
relationships (Scheme 2). In this two-step reversible reaction scheme, the activated sorption of A to form $\mathrm{B}^{*}$ precedes the activated desorption of $\mathrm{B}^{*}$ to form the final product $\mathrm{B}$. The complexity of the analytical solution for the time-averaged reaction rate for this system renders it challenging to extract key relations between the catalyst LFE properties, the reaction conditions, and the dynamic catalysis rate. We therefore consider simplifying limiting cases which permit derivation of closed-form solutions and, in doing so, facilitate the development of generalizable physical insights regarding the mechanism(s) of rate and conversion enhancement in dynamic catalysis.

Scheme 2. Reversible two-step reaction sequence for the conversion of A to B.

$$
\begin{gathered}
\mathrm{A}+* \rightleftarrows \mathrm{B}^{*} \\
\mathrm{~B}^{*} \rightleftarrows \mathrm{B}+* \\
\text { Overall: } \mathrm{A} \Leftrightarrow \mathrm{B}
\end{gathered}
$$

\subsubsection{Derivation of the dynamic equilibrium constant}

Periodic forced oscillation of rate constants during dynamic catalysis provide an input of thermodynamic work which, akin to biological molecular pumps [26,28-30], can drive chemical reactions beyond the thermodynamic equilibrium limit which prescribes an equilibrium ratio of product and reactant activities, (e.g., $\left.a_{\mathrm{B}}^{\mathrm{eq}} / a_{\mathrm{A}}^{\mathrm{eq}}\right)$, defined by the equilibrium constant, $K=\exp \left(-\Delta G^{\mathrm{o}} / R T\right)$, where $\Delta G^{\mathrm{o}}$ is the standard-state free energy difference between the product and reactants. In the absence of forced kinetic oscillations, the net rate of conversion of reactants to products is zero at thermodynamic equilibrium and the chemical potentials of the reactants and products are equal. We define an analogous "dynamic equilibrium" condition for which the net rate of the overall reaction is zero and the corresponding "dynamic equilibrium" constant, $K_{\mathrm{dyn}}$, is the quotient of reactant and product chemical activities. Unlike $K$, the dynamic equilibrium constant $K_{\mathrm{dyn}}$ is not definable in terms of $\Delta G^{\circ}$, but, rather, is determined by reaction kinetics, as is demonstrated next. 
At dynamic equilibrium for a single-path reaction sequence (e.g. Scheme 2), the time-averaged net rate for each reaction step is zero, such that the difference between the forward and reverse time-averaged rates of elementary steps is zero (eqs. (3) and (4)):

$$
\begin{aligned}
& \left\langle r_{1}\right\rangle-\left\langle r_{-1}\right\rangle=\frac{\int_{t}^{t+\lambda} k_{1}\left(t^{\prime}\right) a_{\mathrm{A}}^{\mathrm{eq}} \theta_{*}\left(t^{\prime}\right) \mathrm{d} t^{\prime}}{\int_{t}^{t+\lambda} \mathrm{d} t^{\prime}}-\frac{\int_{t}^{t+\lambda} k_{-1}\left(t^{\prime}\right) \theta_{\mathrm{B}^{*}}\left(t^{\prime}\right) \mathrm{d} t^{\prime}}{\int_{t}^{t+\lambda} \mathrm{d} t^{\prime}}=0 \\
& \left\langle r_{2}\right\rangle-\left\langle r_{-2}\right\rangle=\frac{\int_{t}^{t+\lambda} k_{2}\left(t^{\prime}\right) \theta_{\mathrm{B}^{*}}\left(t^{\prime}\right) \mathrm{d} t^{\prime}}{\int_{t}^{t+\lambda} \mathrm{d} t^{\prime}}-\frac{\int_{t}^{t+\lambda} k_{-2}\left(t^{\prime}\right) a_{\mathrm{B}}^{\mathrm{eq}} \theta_{*}\left(t^{\prime}\right) \mathrm{d} t^{\prime}}{\int_{t}^{t+\lambda} \mathrm{d} t^{\prime}}=0
\end{aligned}
$$

where $\lambda$ is the wavelength of the oscillation. To facilitate the derivation of simple, interpretable analytical solutions, we consider the limiting case wherein the kinetic oscillation timescale (i.e. $\lambda$ ) is much smaller than the characteristic timescales for the fractional coverages to change and, thusly, the fractional coverages are approximated as time-invariant, i.e., $\theta_{\mathrm{B}^{*}}(t) \approx \theta_{\mathrm{B}^{*}}$, such that eqs. (3) and (4) reduce to (eqs. (5) and (6)):

$$
\begin{aligned}
& \left\langle r_{1}\right\rangle-\left\langle r_{-1}\right\rangle=a_{\mathrm{A}}^{\mathrm{eq}} \theta_{*}^{\mathrm{eq}} \frac{\int_{t}^{t+\lambda} k_{1}\left(t^{\prime}\right) \mathrm{d} t^{\prime}}{\int_{t}^{t+\lambda} \mathrm{d} t^{\prime}}-\theta_{\mathrm{B}^{*}}^{\mathrm{eq}} \frac{\int_{t}^{t+\lambda} k_{-1}\left(t^{\prime}\right) \mathrm{d} t^{\prime}}{\int_{t}^{t+\lambda} \mathrm{d} t^{\prime}}=\left\langle k_{1}\right\rangle a_{\mathrm{A}}^{\mathrm{eq}} \theta_{*}^{\mathrm{eq}}-\left\langle k_{-1}\right\rangle \theta_{\mathrm{B}^{*}}^{\mathrm{eq}}=0 \\
& \left\langle r_{2}\right\rangle-\left\langle r_{-2}\right\rangle=\theta_{\mathrm{B}^{*}}^{\mathrm{eq}} \frac{\int_{t}^{t+\lambda} k_{2}\left(t^{\prime}\right) \mathrm{d} t^{\prime}}{\int_{t}^{t+\lambda} \mathrm{d} t^{\prime}}-a_{\mathrm{B}}^{\mathrm{eq}} \theta_{*}^{\mathrm{eq}} \frac{\int_{t}^{t+\lambda} k_{-2}\left(t^{\prime}\right) \mathrm{d} t^{\prime}}{\int_{t}^{t+\lambda} \mathrm{d} t^{\prime}}=\left\langle k_{2}\right\rangle \theta_{\mathrm{B}^{*}}^{\mathrm{eq}}-\left\langle k_{-2}\right\rangle a_{\mathrm{B}}^{\mathrm{eq}} \theta_{*}^{\mathrm{eq}}=0
\end{aligned}
$$

where brackets, “〈 $\rangle$ ”, indicate time-averaged quantities. We now define the dynamic equilibrium constant, analogous to the thermodynamic equilibrium constant, by rearrangement of eqs. (5) and (6) to yield the ratio of $a_{\mathrm{B}} / a_{\mathrm{A}}$ as (eq. (7)):

$$
K_{\text {dyn }}=\frac{a_{\mathrm{B}}^{\mathrm{eq}}}{a_{\mathrm{A}}^{\mathrm{eq}}}=\frac{\left\langle k_{1}\right\rangle\left\langle k_{2}\right\rangle}{\left\langle k_{-1}\right\rangle\left\langle k_{-2}\right\rangle}
$$


In general, for single-path reactions at quasi-static surface conditions, the dynamic equilibrium constant is a product of $\left\langle k_{j}\right\rangle /\left\langle k_{-j}\right\rangle$ raised to the power of the stoichiometric number of that reaction step, which is unity for both reactions in Scheme 2. Under a forced square-wave oscillation, each $\left\langle k_{j}\right\rangle$ is the average of $k_{j}^{[n]}$ weighted by the duration, $\delta t^{[n]}$, spent in each kinetic state $n$ (see Scheme 1) (eq. (8)):

$$
\begin{gathered}
\left\langle k_{j}\right\rangle=\frac{\int_{0}^{\delta t^{[1]}+\delta t^{[2]}} k_{j}\left(t^{\prime}\right) \mathrm{d} t^{\prime}}{\int_{0}^{\delta t^{[1]}+\delta t^{[2]}} \mathrm{d} t}=\frac{\int_{0}^{\delta t^{[1]}} k_{j}^{[1]} \mathrm{d} t^{\prime}+\int_{\delta t^{[1]}}^{\delta t^{[1]}}+\delta t^{[2]} k_{j}^{[2]} \mathrm{d} t^{\prime}}{\int_{0}^{\delta t^{[1]}+\delta t^{[2]}} \mathrm{d} t}=\frac{\delta t^{[1]} k_{j}^{[1]}+\delta t^{[2]} k_{j}^{[2]}}{\delta t^{[1]}+\delta t^{[2]}} \\
=\frac{k_{j}^{[1]}}{1+\delta t^{[2]} / \delta t^{[1]}}\left(1+\frac{\delta t^{[2]}}{\delta t^{[1]}} \frac{k_{j}^{[2]}}{k_{j}^{[1]}}\right)
\end{gathered}
$$

Substitution of eq. (8) into eq. (7) gives (eq. (9)):

$$
K_{\mathrm{dyn}}=\frac{k_{1}^{[1]} k_{2}^{[1]}}{k_{-1}^{[1]} k_{-2}^{[1]}} \frac{\left(1+\frac{\delta t^{[2]}}{\delta t^{[1]}} \frac{k_{1}^{[2]}}{k_{1}^{[1]}}\right)\left(1+\frac{\delta t^{[2]}}{\delta t^{[1]}} \frac{k_{2}^{[2]}}{k_{2}^{[1]}}\right)}{\left(1+\frac{\delta t^{[2]}}{\delta t^{[1]}} \frac{k_{-1}^{[2]}}{k_{-1}^{[1]}}\right)\left(1+\frac{\delta t^{[2]}}{\delta t^{[1]}} \frac{k_{2}^{[2]}}{k_{-2}^{[1]}}\right)}
$$

for which the leading term, $k_{1}^{[1]} k_{2}^{[1]} / k_{-1}^{[1]} k_{-2}^{[1]}$, is equal to the thermodynamic equilibrium constant, $K$. Each rate constant ratio in eq. (9), $k_{j}^{[2]} / k_{j}^{[1]}$, can be re-expressed in terms of the variation, from state [1] to state [2], of the free energy of activation for the corresponding step (eq. (10)):

$$
\frac{k_{j}^{[2]}}{k_{j}^{[1]}}=\exp \left(-\frac{\Delta \Delta G_{j}^{\text {of }}}{R T}\right)
$$

where $\Delta \Delta G_{j}^{\mathrm{o \ddagger}} \equiv \Delta G_{j}^{\mathrm{o} \ddagger[2]}-\Delta G_{j}^{\mathrm{o \ddagger}[1]}$. In reaction sequences described by linear free energy (LFE) relationships, the oscillation amplitudes for the free energies of activation of each elementary step, $\Delta \Delta G_{j}^{\mathrm{o} \ddagger}$, 
are not independent of each other, but are constrained by a scaling parameter. Here, we define free-energy scaling parameters in terms of $\Delta \Delta G_{2}^{\text {oł }}$, such that (eq. (11)):

$$
\omega_{j} \equiv \frac{\Delta \Delta G_{j}^{\mathrm{o} \ddagger}}{\Delta \Delta G_{2}^{\mathrm{of}}}
$$

where $\omega_{2}=1$ by definition. In this work, $\omega_{j}$ are constants, but their definition in eq. (11) only concerns the relative change in free energies of activation with respect to each other; $\omega_{j}$ can, in general, vary as a function of the oscillation endpoints if scaling relationships are non-linear. Description of Scheme 2 in terms of LFE relationships is completed by recognizing that the free energy change of the overall reaction is constant, and thus the set of $\omega_{j}$ is not linearly independent (eq. (12)):

$$
\begin{aligned}
& \Delta G_{\mathrm{ovr}}^{\mathrm{o}}=\sum_{\mathrm{fwd}} \Delta G_{j}^{\mathrm{o} \ddagger}-\sum_{\mathrm{rvs}} \Delta G_{j}^{\mathrm{of}} \\
& \frac{\Delta \Delta G_{\mathrm{ovr}}^{\mathrm{o}}}{\Delta \Delta G_{2}^{\mathrm{o} \ddagger}}=0=\sum_{\mathrm{fwd}} \omega_{j}-\sum_{\mathrm{rvs}} \omega_{j}
\end{aligned}
$$

where the difference between $\omega_{j}$ in the forward and reverse directions on a reaction path must sum to zero. The constraints provided by eq. (12) enable description of the reaction in Scheme 2 in exclusive terms of $\omega_{1}$ and $\omega_{-1}$ by recognizing $\omega_{2}=1$ and $\omega_{-2}=\omega_{1}+\omega_{2}-\omega_{-1}=1+\omega_{1}-\omega_{-1}$. After substitution of these expressions for $\omega_{-2}$ and $\omega_{2}$ with eqs. (10) and (11) into eq. (9), $K_{\mathrm{dyn}}$ is obtained in terms of $\Delta \Delta G_{2}^{o \neq}$, the asymmetry of the square-wave oscillation $\left(\delta t^{[2]} / \delta t^{[1]}\right)$, and scaling parameters $\left(\omega_{1}\right.$ and $\left.\omega_{-1}\right)$ (eq. (13)): 


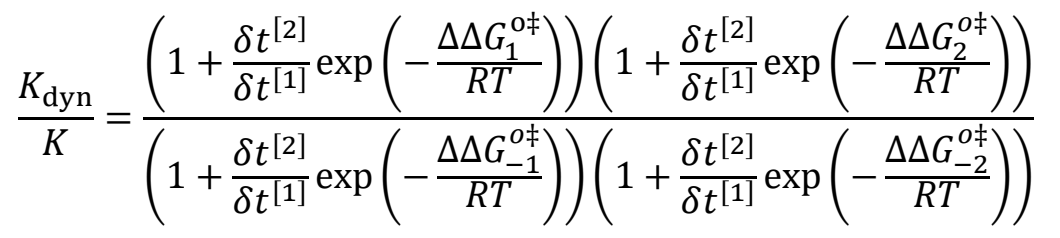

$$
\begin{aligned}
& =\frac{\left(1+\frac{\delta t^{[2]}}{\delta t^{[1]}} \exp \left(-\omega_{1} \frac{\Delta \Delta G_{2}^{o \ddagger}}{R T}\right)\right)\left(1+\frac{\delta t^{[2]}}{\delta t^{[1]}} \exp \left(-\frac{\Delta \Delta G_{2}^{o \ddagger}}{R T}\right)\right)}{\left(1+\frac{\delta t^{[2]}}{\delta t^{[1]}} \exp \left(-\omega_{-1} \frac{\Delta \Delta G_{2}^{o \ddagger}}{R T}\right)\right)\left(1+\frac{\delta t^{[2]}}{\delta t^{[1]}} \exp \left(-\left(1+\omega_{1}-\omega_{-1}\right) \frac{\Delta \Delta G_{2}^{o \ddagger}}{R T}\right)\right)}
\end{aligned}
$$

In eq. (13), $\delta t^{[2]} / \delta t^{[1]}$ and $\Delta \Delta G_{2}^{o \ddagger}$ are controllable quantities of the kinetic oscillation, while $\omega_{j}$ are properties of the catalyst. We emphasize that, at quasi-static conditions, $K_{\text {dyn }}$ (eq. (13)) is not a function of $\Delta G_{2}^{\mathrm{o} \neq[i]}$ in either state; $K_{\mathrm{dyn}}$ is entirely determined by the amplitude of the oscillation, scaling parameters, and asymmetry of oscillation.

By inspection of eq. (13), we identify critical catalyst properties, in terms of $\omega_{j}$, that determine the behavior of $K_{\mathrm{dyn}}$ and the reaction rate at reversible conditions (vide infra). The first condition, $\omega_{1}=\omega_{-1}$, for which $K_{\mathrm{dyn}}=K$, is an "iso-equilibrium" line. At these conditions, $k_{1}^{[2]} / k_{1}^{[1]}=k_{-1}^{[2]} / k_{-1}^{[1]}$ and $k_{2}^{[2]} / k_{2}^{[1]}=k_{-2}^{[2]} / k_{-2}^{[1]}$, thus cancelling out in eq. (9). A second iso-equilibrium line is identified as $\omega_{-1}=$ 1 , where $k_{1}^{[2]} / k_{1}^{[1]}=k_{-2}^{[2]} / k_{-2}^{[1]}$ and $k_{-1}^{[2]} / k_{-1}^{[1]}=k_{2}^{[2]} / k_{2}^{[1]}$, and thus again cancel out. Three more linear relationships of $\omega_{1}$ and $\omega_{-1}$ determine whether correlations between each $\Delta G_{j}^{\text {of }}$ with $\Delta G_{2}^{\text {of }}$ are negative or positive: $\omega_{1}=0, \omega_{-1}=0$, and $\omega_{2}=1+\omega_{1}-\omega_{-1}=0$. The signs of $\omega_{1}, \omega_{-1}$ and $\omega_{-2}$ define the behavior of $K_{\mathrm{dyn}}$ because the terms $1+\exp (a x)$ in eq. (13) approach unity $(a<0)$ or infinity $(a>0)$ at large $x$; thus, the signs of the coefficients that multiply $\Delta \Delta G_{2}^{o \ddagger}$ in eq. (13) determine whether $K_{\text {dyn }}$ approaches zero, infinity, or $K$ as $\Delta \Delta G_{2}^{\text {oł }} \rightarrow \infty$. We illustrate the kinetic insight and clarity proffered by these conditions by inspection of $K_{\text {dyn }}$ as a function of $\omega_{1}$ and $\omega_{-1}$ at oscillation amplitudes $\Delta \Delta G_{2}^{\text {of }} / R T$ of 0.01 and 100 (Figure 1). We note that eq. (13) is symmetric about $\Delta \Delta G_{2}^{\text {of }}=0$, and thus we take $\Delta \Delta G_{2}^{\text {oł }}>$ 
0 as convention. The solid lines in Figure 1 defines where $\omega_{1}, \omega_{-1}$ or $\omega_{-2}$ change signs and each dashed line is an iso-equilibrium condition where the effects of oscillating each $\Delta G_{j}^{\mathrm{o \ddagger}}$ cancel $\left(\omega_{1}=\omega_{-1}\right.$ and $\omega_{-1}=$ 1). At low oscillation amplitudes (Figure $1 \mathrm{a}), K_{\text {dyn }}>K$ if the catalyst properties $\omega_{j}$ fall between the isoequilibrium lines, and $K_{\mathrm{dyn}}<K$ otherwise. As the oscillation amplitude increases (Figure 1b), the magnitude of the $K_{\mathrm{dyn}} / K$ ratio increases, but now $K_{\mathrm{dyn}} \gg K$ only if $\omega_{j}$ fall between the iso-equilibrium lines and $\omega_{1}<0$. For $\omega_{j}$ that fall in the top right green region of Figure $1 \mathrm{~b}, K_{\mathrm{dyn}} \sim K$ at large oscillation amplitudes but is slightly below thermodynamic equilibrium $\left(K_{\text {dyn }} \lesssim K\right)$ outside of the iso-equilibrium lines and slightly above $\left(K_{\mathrm{dyn}} \gtrsim K\right)$ for $\omega_{j}$ between the iso-equilibrium lines.

Figure 1a and Figure 1b show the dynamic equilibrium constants for symmetric square waveforms; however, as we previously detailed [16], asymmetry in oscillation waveform is a potent lever for enhancing the reaction rate and driving dynamic equilibrium beyond the thermodynamic limit. The optimal temporal asymmetry in the oscillation square wave is found by taking the derivative of eq. (13) with respect to $\delta t^{[2]} / \delta t^{[1]}$ and equating $\mathrm{d} K_{\mathrm{dyn}} / \mathrm{d}\left(\delta t^{[2]} / \delta t^{[1]}\right)$ to zero. There is one positive $\delta t^{[2]} / \delta t^{[1]}$ solution to this equation (the negative solution is non-physical), which corresponds to the extremum of $K_{\mathrm{dyn}}$. The extremum in $K_{\mathrm{dyn}}$ as a function of $\delta t^{[2]} / \delta t^{[1]}$ is located at (eq. (14)):

$$
\left(\frac{\delta t^{[2]}}{\delta t^{[1]}}\right)_{\mathrm{ext}}=\exp \left(\frac{\omega_{1}+1}{2} \frac{\Delta \Delta G_{2}^{o \ddagger}}{R T}\right)
$$

where "extremum" is abbreviated to "ext" in the subscript. Evaluating $K_{\mathrm{dyn}}$ at the extremum condition by substitution of eq. (14) into eq. (13) gives the results shown in Figure 1c. At quasi-static conditions with square-wave asymmetry corresponding to the extremum, $K_{\mathrm{dyn}}>K$ for all $\omega_{j}$ between the iso-equilibrium lines, and $K_{\mathrm{dyn}}<K$ outside of the iso-equilibrium lines. Between these lines, $K_{\mathrm{dyn}}$ is driven to infinity by increasing the oscillation amplitude $\Delta \Delta G_{2}^{o \ddagger}$ and adjusting $\delta t^{[1]}$ and $\delta t^{[2]}$ in accordance to eq. (14). The best 
dynamic catalysts have $\omega_{1}$ that deviate significantly from unity and $\omega_{-1}=\left(\omega_{1}+1\right) / 2$, which is the line that bisects the iso-equilibrium lines and is the maximum in $K_{\mathrm{dyn}}$ as a function of $\omega_{-1}$.

The analysis thus far of the simple, two-step reaction scheme provides critical insights into the necessary relationships between free energies of activation to achieve supra-equilibrium conversion with high frequency oscillations (quasi-static conditions). From closed-form formulation of the dynamic equilibrium constant, we see that each reaction step $j$ contributes a term in the form $1+$ $\delta t^{[2]} / \delta t^{[1]} \exp \left(-\Delta \Delta \mathrm{G}_{j}^{o \ddagger} / R T\right)$ raised to the power of the stoichiometric number $+\sigma_{j}$ for the forward reaction and $-\sigma_{j}$ for the reverse reaction. By inspection of this function, iso-equilibrium lines where $K_{\mathrm{dyn}}=K$ are identified; these iso-equilibrium lines bound the regions that define the limiting behavior of $K_{\text {dyn }}$ (Figure 1c). Specifically, for this system, optimal conversion is achieved for catalysts that fall between the iso-equilibrium lines. For catalysts that fall between the iso-equilibrium lines and have $\omega_{1}<0$ - where the activation free energy for the first forward step is negatively correlated with that for the second forward step-the symmetric square wave oscillation gives $K_{\mathrm{dyn}} \rightarrow \infty$ as the oscillation amplitude increases to infinity, enabling $100 \%$ conversion of any reactants. 


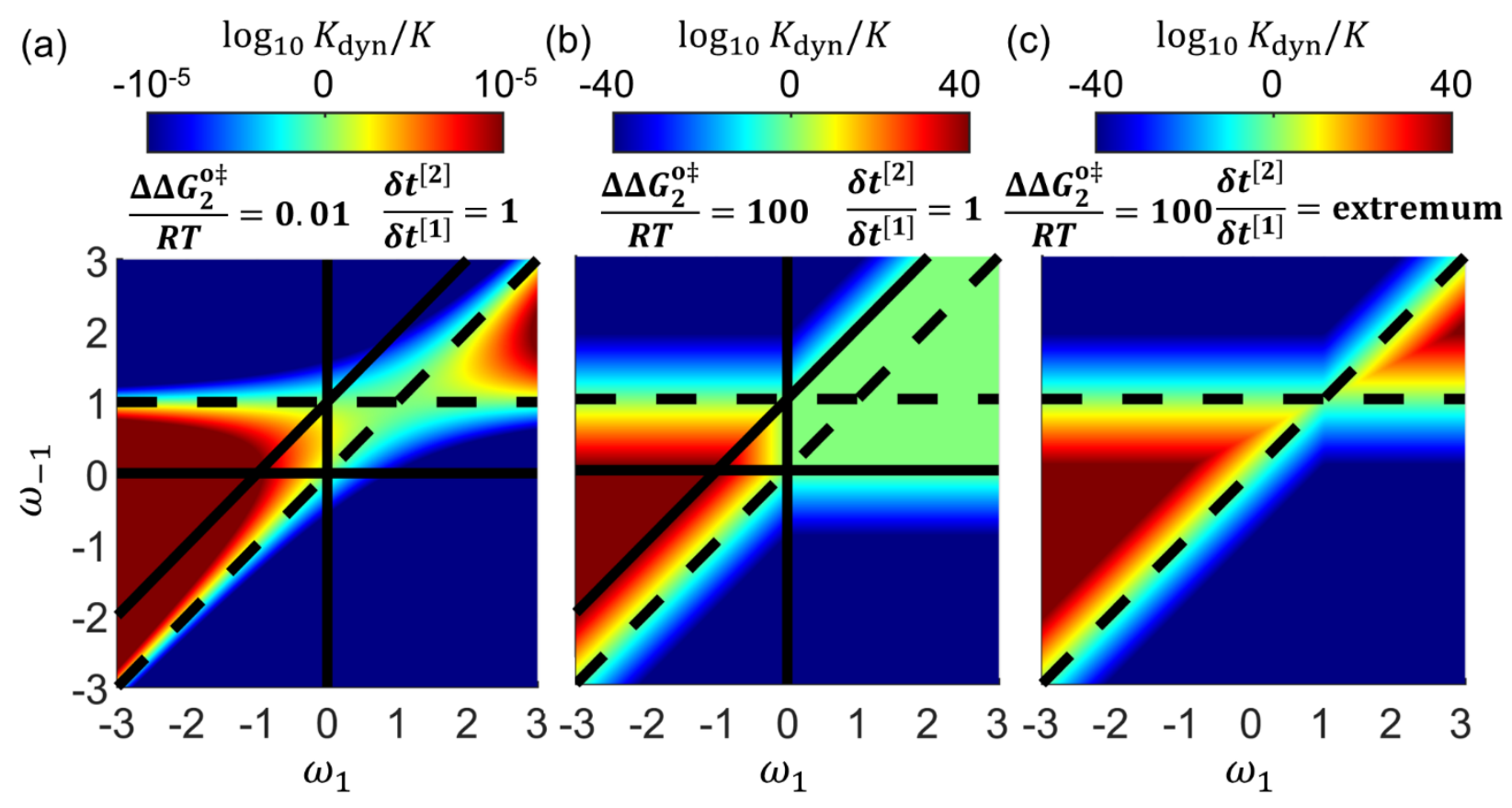

Figure 1. Dynamic equilibrium constant (eq. (13)) with oscillation amplitudes and square-wave symmetry (a) $\Delta \Delta G_{2}^{o \ddagger}=0.01 R T, \delta t^{[2]} / \delta t^{[1]}=1$ (b) $\Delta \Delta G_{2}^{o \ddagger}=100 R T, \delta t^{[2]} / \delta t^{[1]}=1$, and (c) $\Delta \Delta G_{2}^{o \ddagger}=100 R T$, $\delta t^{[2]} / \delta t^{[1]}=\left(\delta t^{[2]} / \delta t^{[1]}\right)$ ext (see eq. (13)), which is the minimum or maximum of $K_{\text {dyn }}$ as a function of $\delta t^{[2]} / \delta t^{[1]}$.

\subsubsection{Derivation of the time-averaged dynamic reaction rate}

The discussion above detailed the exploration of the ideal properties for achieving maximum conversion of reactants to products, as quantified by the dynamic equilibrium constant, $K_{\mathrm{dyn}}$. Following a similar analysis, we next explicate the ideal catalyst properties for enhanced reaction rates at quasi-static surface conditions with respect to the Sabatier maximum static catalysis reaction rate. In static catalysis, the rate of the two-step reaction in Scheme 2 is given as (eq. (15)):

$$
r=\frac{k_{1} a_{\mathrm{A}} k_{2}-k_{-1} k_{-2} a_{\mathrm{B}}}{k_{1} a_{\mathrm{A}}+k_{2}+k_{-1}+k_{-2} a_{\mathrm{B}}}
$$

Each $k_{j}$ is a function of $\Delta G_{2}^{\text {oł }}$ which is adjusted to maximize $r$; this is the Sabatier maximum. Each $k_{j}$ is given as (eq. (16)): 


$$
k_{j}=k_{j, 0} \exp \left(-\omega_{j} \frac{\Delta G_{2}^{\text {oł }}}{R T}\right)
$$

where $k_{j, 0}$ is a pre-exponential factor defined as $k_{j}$ at $\Delta G_{2}^{\text {of }}=0$. An analytical solution for the Sabatier maximum does not exist for the general case shown in eq. (15), requiring numerical solvers to find the location and value of the peak [31]. The dynamic catalysis rate is significantly more complicated, and thus an analytical solution for the maximum dynamic catalysis rate is also out of reach, but a closed-form function for the time-averaged rate is found by recognizing that under quasi-static surface conditions, the time-averaged rate constants $\left\langle k_{j}\right\rangle$ replace $k_{j}$ in all in the mass-action rate equations; thus, the dynamic catalysis rate function is simply the static catalysis rate function with $\left\langle k_{j}\right\rangle$ substituted for $k_{j}$ (eq. (17)):

$$
\langle r\rangle=\frac{\left\langle k_{1}\right\rangle a_{\mathrm{A}}\left\langle k_{2}\right\rangle-\left\langle k_{-1}\right\rangle\left\langle k_{-2}\right\rangle a_{\mathrm{B}}}{\left\langle k_{1}\right\rangle a_{\mathrm{A}}+\left\langle k_{2}\right\rangle+\left\langle k_{-1}\right\rangle+\left\langle k_{-2}\right\rangle a_{\mathrm{B}}}
$$

where $\left\langle k_{j}\right\rangle$ in terms of scaling parameters are (eq. (18)):

$$
\left\langle k_{j}\right\rangle \equiv \frac{\delta t^{[1]} k_{j}^{[1]}+\delta t^{[2]} k_{j}^{[2]}}{\delta t^{[1]}+\delta t^{[2]}}=\frac{k_{j, 0} \exp \left(-\omega_{j} \frac{\Delta G_{2}^{o \ddagger[1]}}{R T}\right)+k_{j, 0} \frac{\delta t^{[2]}}{\delta t^{[1]}} \exp \left(-\omega_{j} \frac{\Delta G_{2}^{o \ddagger[2]}}{R T}\right)}{1+\delta t^{[2]} / \delta t^{[1]}}
$$

Unlike the dynamic equilibrium constant, the dynamic rate is a function of the factors $k_{j, 0}$ and the trough $\left(\Delta G_{2}^{\mathrm{o \ddagger}[1]}\right)$ and peak $\left(\Delta G_{2}^{\mathrm{o \ddagger}}{ }^{[2]}\right)$ of the oscillation wave, and not simply the amplitude $\left(\Delta \Delta G_{2}^{o \ddagger}\right)$. For an arbitrary choice of $k_{j, 0}=1$, the time-averaged rate depends on three parameters for each set of $\omega_{j}$ $\left(\Delta G_{2}^{o \ddagger[1]}, \Delta G_{2}^{o \ddagger[2]}\right.$ and $\left.\delta t^{[2]} / \delta t^{[1]}\right)$-resulting in a description of rate for which we employ a mixed optimization-brute force approach (see Section 2) to find the global maximum dynamic catalysis rate. The ratio of the maximum dynamic and static catalysis rates are shown in Figure 2a for a completely irreversible reaction $\left(a_{\mathrm{A}}=1, a_{\mathrm{B}}=0\right)$ and in Figure $1 \mathrm{~b}$ for weakly reversible conditions that are still far from thermodynamic equilibrium $\left(a_{\mathrm{A}}=1, a_{\mathrm{B}}=0.05\right)$. In Figure $2 \mathrm{a}$, the irreversible reaction is enhanced 
significantly only if $\omega_{1}<0$, which is where the activation free energy of the first reaction step is negatively correlated with that of the second reaction step. In the presence of a small amount of product, the benefits of dynamic catalysis for $\omega_{-1}>1$ and for $\omega_{-1}<\omega_{1}$ disappear (Figure $2 b$ ). For $\omega_{-1}>1, \omega_{1}<0$, the oscillation amplitude $\Delta \Delta G_{-2}^{o \ddagger}$ correlates positively with and exceeds the oscillation amplitude $\Delta \Delta G_{1}^{o \ddagger}$, while $\Delta \Delta G_{-1}^{o \ddagger}$ correlates positively with and exceeds the magnitude of $\Delta \Delta G_{2}^{o \ddagger}$, which lowers the dynamic equilibrium constant (eq. (13)). This lowering of $K_{\mathrm{dyn}}$ results in driving the reaction in the reverse direction in the presence of a small amount of product, thus limiting the overall conversion. Similarly, dynamic catalysts below the $\omega_{-1}=\omega_{1}$ line are also hindered by the decrease in $K_{\mathrm{dyn}}$. Thus, a dynamic catalyst is functionally useless if it decreases $K_{\text {dyn }}$; while it may enhance the rate in the absence of product (Figure 2a), any amount of B hinders the reaction to such an extent that the dynamic equilibrium constant is orders of magnitude lower than the thermodynamic equilibrium constant (Figure 2b). For the reaction in Scheme 2, a good dynamic catalyst for enhancing the reaction rate under quasi-static conditions falls between the iso-equilibrium lines and has $\omega_{1}<0$. While the catalysts that fall between the iso-equilibrium lines with $\omega_{1}>0$ achieve supra-equilibrium conversion by asymmetry of the square wave oscillation, this effects little rate enhancements at irreversible conditions when all $k_{j, 0}=1$. However, as shown in Figure $2 \mathrm{c}$, the maximum dynamic catalysis reaction rate at thermodynamic equilibrium $\left(a_{\mathrm{A}}=a_{\mathrm{B}}=1\right)$ is positive for all catalysts that fall between the iso-equilibrium lines. 
(a)

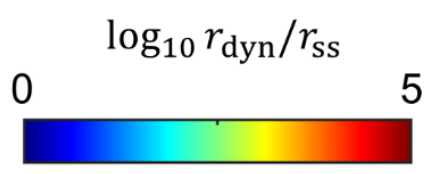

$$
a_{\mathrm{A}}=1, a_{\mathrm{B}}=0
$$

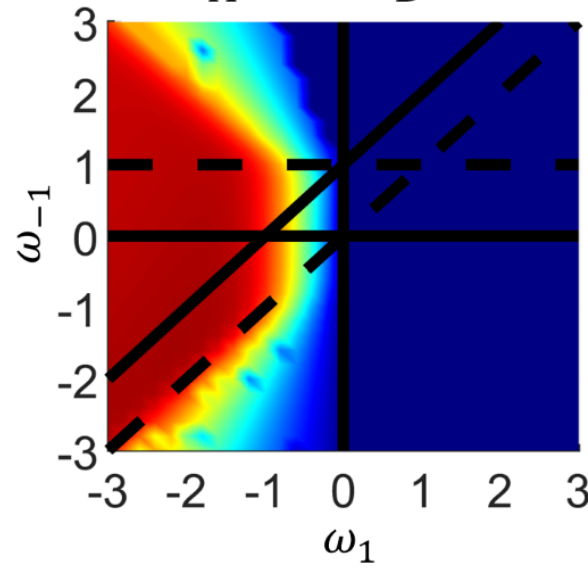

(b)

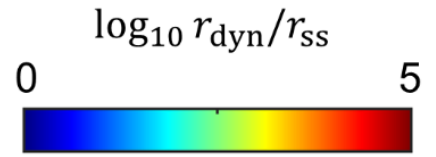

$a_{\mathrm{A}}=1, a_{\mathrm{B}}=0.05$

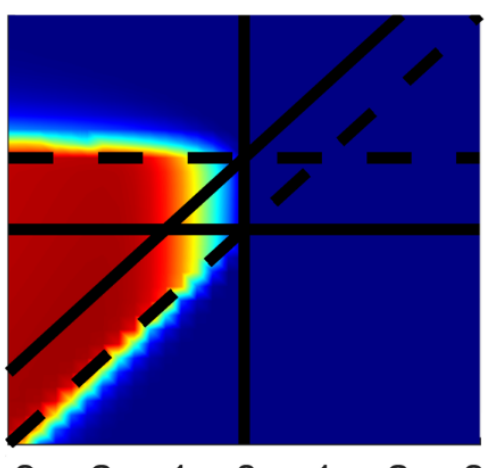

$\omega_{1}$ (c)

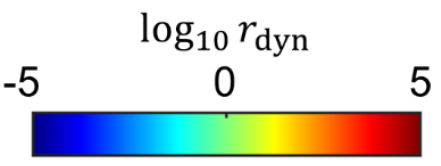

$a_{\mathrm{A}}=a_{\mathrm{B}}=1$

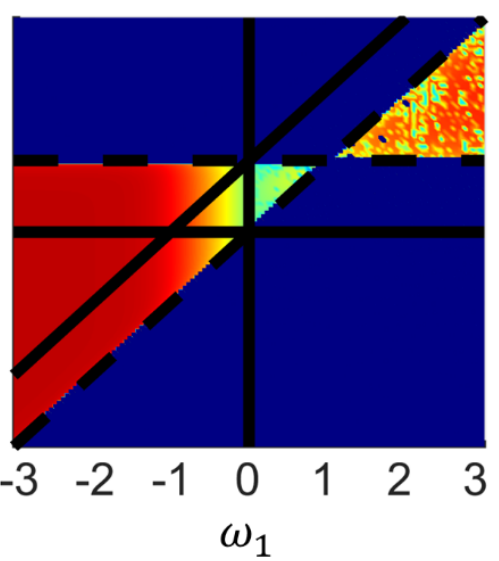

Figure 2. The ratio of the maximum dynamic catalysis rate $\left(r_{\mathrm{dyn}}\right)$ and the Sabatier maximum steady-state static catalysis rate $\left(r_{\mathrm{SS}}\right)$ as a function of the linear scaling parameters $\omega_{j}$ at (a) $a_{\mathrm{A}}=1, a_{\mathrm{B}}=0$ (b) $a_{\mathrm{A}}=$ $1, a_{\mathrm{B}}=0.05$ (c) $a_{\mathrm{A}}=1, a_{\mathrm{B}}=1$ (thermodynamic equilibrium). The maximum dynamic catalysis rates were found by brute-force searches of kinetic oscillation waveshapes by systematically varying $\Delta G_{2}^{o \ddagger[1]}$, $\Delta G_{2}^{o \ddagger[2]}$ and $\delta t^{[2]} / \delta t^{[1]}$ in eqs. (17) and (18) followed by convergence to a local minimum using the builtin Matlab function fminunc. The Sabatier maximum was found by using fminunc to maximize the rate by varying $\Delta G_{2}^{\mathrm{o} \ddagger}$. The reference $k_{j, 0}$ were arbitrarily set equal to unity. Artifacts in the figures are a result of the brute-force method converging to different local maximums.

Comparison of Figure 1 and Figure 2 reveals the close relationship between $K_{\text {dyn }}$ and $r_{\text {dyn }}$. The dynamic equilibrium constant has a closed-form solution (eq. (13)) which proffers insights into the key parameters necessary for enhancing $K_{\mathrm{dyn}}$, and these insights generally extend to $r_{\mathrm{dyn}}$. Thus far, we have exclusively considered only oscillation frequencies much faster than the time scales for chemical transformations, where the quasi-static surface approximation $\left(\theta_{j^{*}}(t) \approx \theta_{j^{*}}\right)$ is valid. However, it has been shown that some kinetic systems do not exhibit rate enhancement at the high frequency limit, but, rather, are only appreciably accelerated within a band of finite "resonance" frequencies bound by characteristic relaxation times associated with the reaction kinetics. The word "resonance" is here within quotation marks because the concerned phenomenon is not the same as mechanical resonance- the dynamic behavior in the systems discussed herein do not exhibit any natural resonant frequency and the eigenvalues of the dynamic 
systems discussed in this work are real numbers, precluding any unforced oscillatory behavior by the kinetic systems themselves. Nonetheless, we regard "resonance" a useful term for concisely describing this type of dynamic catalysis behavior because of the superficial similarities with well-established mechanical resonance phenomenon. An illustrative comparison of dynamic catalysis resonance and quasi-static behavior are shown in Figure 3.

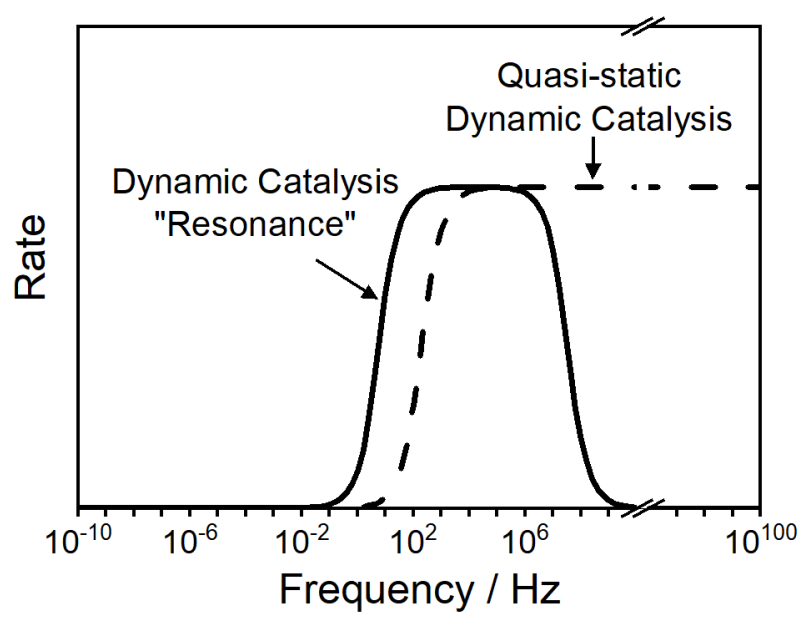

Figure 3. A schematic representation of "resonance" and quasi-static behaviors in dynamic catalysis. "Resonance" is characterized by a finite frequency band that gives enhanced rates of reaction. In quasistatic dynamic catalysis, the oscillation frequencies are sufficiently fast that the chemical activities of all species are nearly time-invariant, and thus there is only a minimum threshold for the frequency. The behavior and required catalyst properties differs for each of these dynamic catalysis mechanisms.

\subsection{Dynamic enhancement of conversion and rate for a three-step reaction sequence-Unifying concepts of resonance and quasi-static dynamic catalysis}

Precedent literature has demonstrated that the manifestation of a resonance frequency band is a consequence of multiple processes occurring during a single kinetic state [16,21]. For the reaction in Scheme 2, there are only two reaction steps: the sorption of $\mathrm{A}^{*}$ and the conversion of $\mathrm{A}^{*}$ to $\mathrm{B}$, each achieved by a separate kinetic state. Thus, no kinetic state is responsible for multiple reaction steps and therefore there are no resonance conditions, as confirmed by a brute-force search for resonance frequencies. In this section, we demonstrate the generality of the approach detailed hereinbefore for a three-step reaction sequence (Scheme 3) that exhibits enhancement to rates and conversions during dynamic catalysis by both resonance and quasi-static mechanisms. 
Scheme 3 shows the three-step reaction sequence that is oft used as an instructive model system for study of dynamic catalysis. In line with previous work exhibiting resonance [18,19,27], the adsorption in all examples considered herein are "barrierless" and not functions of the oscillating parameter, such that $k_{1}^{[2]}=k_{1}^{[1]}=k_{-3}^{[2]}=k_{-3}^{[1]}$. Further, the free energy barrier for the desorption of the product $\mathrm{B}^{*}, \Delta G_{3}^{\mathrm{o} \ddagger}$, is equal to the binding energy of $\mathrm{B}, \mathrm{BE}_{\mathrm{B}}=G_{\mathrm{B}}^{\mathrm{o}}+G_{*}^{\mathrm{o}}-G_{\mathrm{B}^{*}}^{\mathrm{o}}=\Delta G_{3}^{\mathrm{o} \ddagger}$. Thus, we regard $\omega_{j}$ for this reaction sequence to be most conveniently defined as (eq. (19)):

$$
\omega_{j}=\frac{\Delta \Delta \mathrm{G}_{j}^{\mathrm{o} \ddagger}}{\Delta \mathrm{BE}_{\mathrm{B}}}
$$

The reaction in Scheme 3 has been investigated previously by Dauenhauer and coworkers [18] by defining (i) $\gamma \equiv \Delta \mathrm{BE}_{\mathrm{B}} / \Delta \mathrm{BE}_{\mathrm{A}}$ to relate the binding energies of $\mathrm{A}$ and $\mathrm{B}$ and (ii) $\alpha \equiv \Delta E_{\mathrm{a}, \mathrm{sr}} / \Delta H_{\mathrm{sr}}$. to relate the activation energy of the surface reaction $\left(\mathrm{A}^{*} \rightarrow \mathrm{B}^{*}\right)$ to the enthalpy change of the surface reaction. This formalism is equivalent to the $\omega_{j}$ formalism used herein, with $\omega_{-1}=1 / \gamma$ and $\omega_{2}=\alpha(1 / \gamma-1)$. We prefer the $\omega_{j}$ approach here because the salient relationships in dynamic catalysis describe the correlation of free energies of activation rather than energies of transition states and species. We also note that herein we make no consideration on the bounds on the validity of the LFE relationships that arise in the $\alpha-\gamma$ formalism and manifest as negative activation energies (see Section S2 of the Supporting Information).

Scheme 3. Reversible three-step reaction sequence for the conversion of A to B.

$$
\begin{aligned}
& \mathrm{A}+* \rightleftarrows \mathrm{A}^{*} \\
& \mathrm{~A}^{*} \rightleftarrows \mathrm{B}^{*} \\
& \mathrm{~B}^{*} \rightleftarrows \mathrm{B}+* \\
& \text { Overall: } \mathrm{A} \Leftrightarrow \mathrm{B}
\end{aligned}
$$

\subsubsection{Derivation of the dynamic equilibrium constant for the quasi-static regime}

The derivation of $K_{\mathrm{dyn}}$ at quasi-static conditions for the reaction in Scheme 3 follows the same procedure as the derivation of $K_{\mathrm{dyn}}$ (eq. (9)) for the reaction in Scheme 2. $K_{\mathrm{dyn}}$ is thusly (eq. (20)): 


$$
K_{\mathrm{dyn}}=\frac{a_{\mathrm{B}}^{\mathrm{SS}}}{a_{\mathrm{A}}^{\mathrm{ss}}}=\frac{k_{1}^{[1]} k_{2}^{[1]} k_{3}^{[1]}\left(1+\frac{\delta t^{[2]}}{\delta t^{[1]}} \frac{k_{1}^{[2]}}{k_{1}^{[1]}}\right)\left(1+\frac{\delta t^{[2]}}{\delta t^{[1]}} \frac{k_{2}^{[2]}}{k_{2}^{[1]}}\right)\left(1+\frac{\delta t^{[2]}}{\delta t^{[1]}} \frac{k_{3}^{[2]}}{k_{3}^{[1]}}\right)}{k_{-1}^{[1]} k_{-2}^{[1]} k_{-3}^{[1]}\left(1+\frac{\delta t^{[2]}}{\delta t^{[1]}} \frac{k_{-1}^{[2]}}{k_{-1}^{[1]}}\right)\left(1+\frac{\delta t^{[2]}}{\delta t^{[1]}} \frac{k_{-2}^{[2]}}{k_{-2}^{[1]}}\right)\left(1+\frac{\delta t^{[2]}}{\delta t^{[1]}} \frac{k_{-3}^{[2]}}{k_{-3}^{[1]}}\right)}
$$

Now we simplify eq. (20) by dividing both sides of eq. (20) by $K=k_{1}^{[1]} k_{2}^{[1]} k_{3}^{[1]} / k_{-1}^{[1]} k_{-2}^{[1]} k_{-3}^{[1]}$ and recognizing that $k_{1}$ and $k_{-3}$ are both rate constants for barrierless sorption on a surface and thus $k_{1}^{[2]}=$ $k_{1}^{[1]}=k_{-3}^{[2]}=k_{-3}^{[1]}$ to yield eq. $(21)$ :

$$
\begin{aligned}
& \frac{K_{\mathrm{dyn}}}{K}=\frac{\left(1+\frac{\delta t^{[2]}}{\delta t^{[1]}} \frac{k_{2}^{[2]}}{k_{2}^{[1]}}\right)\left(1+\frac{\delta t^{[2]}}{\delta t^{[1]}} \frac{k_{3}^{[2]}}{k_{3}^{[1]}}\right)}{\left(1+\frac{\delta t^{[2]}}{\delta t^{[1]}} \frac{k_{-1}^{[2]}}{k_{-1}^{[1]}}\right)\left(1+\frac{\delta t^{[2]}}{\delta t^{[1]}} \frac{k_{-2}^{[2]}}{k_{-2}^{[1]}}\right)} \\
& =\frac{\left(1+\frac{\delta t^{[2]}}{\delta t^{[1]}} \exp \left(-\frac{\Delta \Delta \mathrm{G}_{2}^{\mathrm{o} \ddagger}}{R T}\right)\right)\left(1+\frac{\delta t^{[2]}}{\delta t^{[1]}} \exp \left(-\frac{\Delta \mathrm{BE}_{\mathrm{B}}}{R T}\right)\right)}{\left(1+\frac{\delta t^{[2]}}{\delta t^{[1]}} \exp \left(-\frac{\Delta \Delta \mathrm{G}_{-1}^{\mathrm{o \ddagger}}}{R T}\right)\right)\left(1+\frac{\delta t^{[2]}}{\delta t^{[1]}} \exp \left(-\frac{\Delta \Delta \mathrm{G}_{-2}^{\mathrm{o \ddagger}}}{R T}\right)\right)} \\
& =\frac{\left(1+\frac{\delta t^{[2]}}{\delta t^{[1]}} \exp \left(-\omega_{2} \frac{\Delta \mathrm{BE}_{\mathrm{B}}}{R T}\right)\right)\left(1+\frac{\delta t^{[2]}}{\delta t^{[1]}} \exp \left(-\frac{\Delta \mathrm{BE}_{\mathrm{B}}}{R T}\right)\right)}{\left(1+\frac{\delta t^{[2]}}{\delta t^{[1]}} \exp \left(-\omega_{-1} \frac{\Delta \mathrm{BE}}{R T}\right)\right)\left(1+\frac{\delta t^{[2]}}{\delta t^{[1]}} \exp \left(-\left(1+\omega_{2}-\omega_{-1}\right) \frac{\Delta \mathrm{BE}_{\mathrm{B}}}{R T}\right)\right)}
\end{aligned}
$$

where the free energy of activation for desorption of $\mathrm{B}$ is equal to the binding energy $\mathrm{BE}_{\mathrm{B}}$. The functional form for $K_{\mathrm{dyn}}$ in the three-step reaction (eq. (21)) is identical to that for the two-step reaction with activated sorption (eq. (13)) after substitution of $\omega_{2}$ for $\omega_{1}$. Correspondingly, $K_{\mathrm{dyn}} / K$ for the three-step reaction sequence is given in Figure 1 with the $\mathrm{x}$-axis changed to $\omega_{2}$.

For the two-step reaction with activated sorption (Section 3.1), it was demonstrated that the behavior of $K_{\mathrm{dyn}} / K$ is closely related to the ratio of the dynamic reaction rate to the Sabatier maximum reaction rate. The relationship extends to the more complex three-step reaction as shown in Figure 4, where supra- 
Sabatier reaction rates are observed at quasi-static conditions for $\omega_{j}$ that fall between the iso-equilibrium lines at (a) irreversible and (b) thermodynamic equilibrium conditions. The derivation of $K_{\mathrm{dyn}} / K$ is simple and straightforward, and thus the intertwined relationship between $K_{\mathrm{dyn}} / K$ and the dynamic reaction rate dramatically simplifies the identification of dynamic catalyst properties that achieve both increases in conversion and reaction rates. In general, the ideal catalyst properties for quasi-static dynamic catalysis are easily found for any single-path reaction sequence.
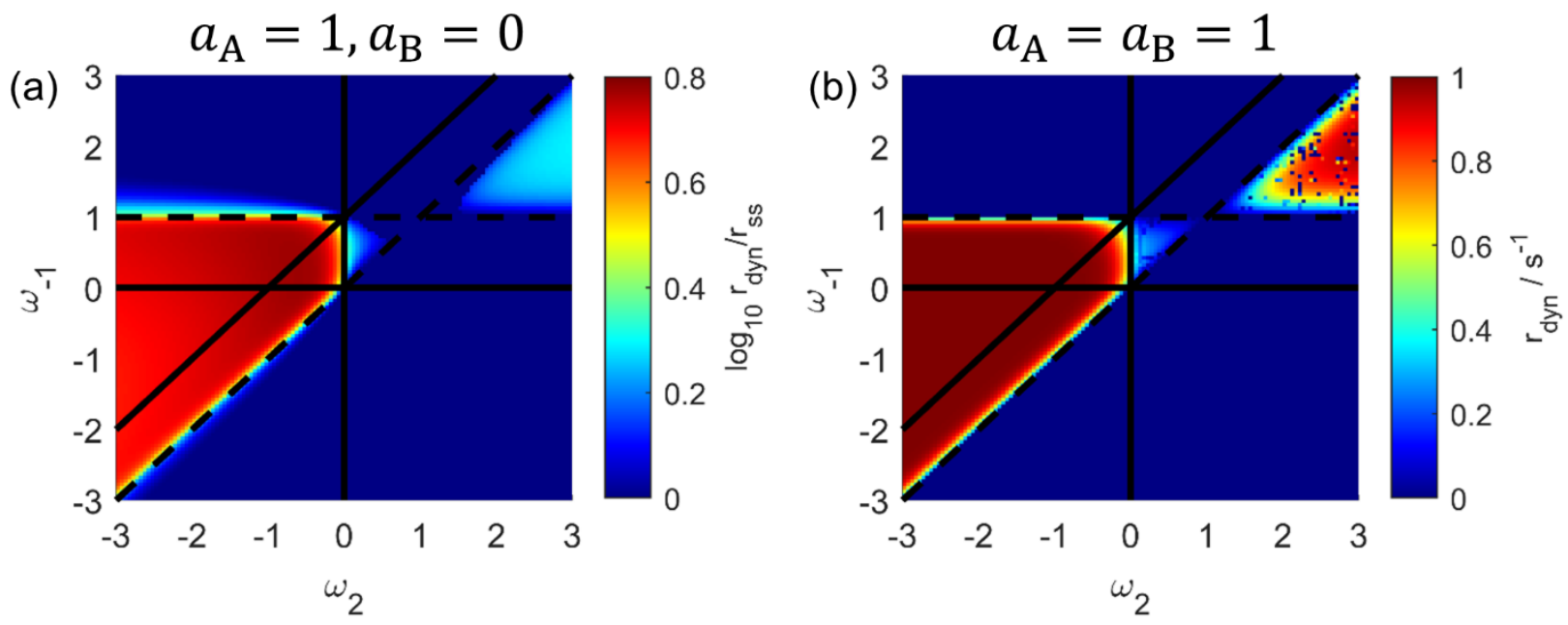

Figure 4. (a) Ratio of the optimal dynamic reaction rate to the steady-state reaction rate as a function of $\omega_{j}$ at irreversible $\left(a_{\mathrm{A}}=1, a_{\mathrm{B}}=0\right)$ reaction conditions. (b) Dynamic reaction rate as a function of $\omega_{j}$ at thermodynamic equilibrium $\left(a_{\mathrm{A}}=a_{\mathrm{B}}=1\right)$. All $k_{j 0}=1 \mathrm{~s}^{-1}$ and $\mathrm{BE}_{\mathrm{B}}^{[j]}$ and $\delta t^{[2]} / \delta t^{[1]}$ were varied at each $\omega_{j}$ to find the optimal dynamic catalysis operating conditions.

In contrast to the simplicity for determining the catalyst properties required for quasi-static dynamic catalysis, identifying the catalyst properties for dynamic catalysis resonance is a greater challenge. In the following section, we explicate the necessary properties for resonance for the three-step reaction sequence in Scheme 3. We demonstrate that while this requires a deeper understanding of the precise dynamic catalysis mechanism, it is still possible to derive closed-forms functions for the reaction rate and dynamic equilibrium constant. 


\subsubsection{Resonance regimes for the three-step reaction sequence}

While eq. (21) and Figure 4 again demonstrate the connection between enhanced conversion and reaction rates during quasi-static dynamic catalysis, previous reports $[18,27]$ have shown that the reaction in Scheme 3, with appropriate LFE scaling parameters, can also exhibit resonance behavior wherein rate and conversion enhancement is confined to a range of finite resonance frequencies.

In our recent work [16], we showed that these ostensible resonance frequencies manifest from the burden of a single kinetic state being responsible for executing multiple elementary steps. In the case of previously reported $[18,27]$ resonance for the reaction in Scheme 3, the kinetic state with low binding of B executes only barrierless desorption steps-recovering B* as product B (step 3) and generating a bare catalyst surface with $\theta_{*}=1$. The second kinetic state, however, is responsible for both adsorbing the reactant A (step 1) and converting $A^{*}$ to $B^{*}$ (step 2). The burden on kinetic state 2 to execute two elementary steps (i) is the origin of apparent "resonant" behavior, with each bounding resonance frequency correspondent to the characteristic timescales of the reaction sequence, and (ii) implies that the ratecontrolling step occurs during kinetic state 2 and determines the time-averaged rate of B formation, $\langle r\rangle$. We therefore surmise that, under optimal "resonant" conditions, $\langle r\rangle$ is at most equal to the rate of B* formation during kinetic state $2, r_{\mathrm{B}^{*}}^{[2]}$, with quasi-equilibrated sorption of A (eq. (22)):

$$
\langle r\rangle \leq r_{\mathrm{B}^{*}}^{[2]}=\frac{k_{2}^{[2]} K_{1}^{[2]} a_{\mathrm{A}}}{1+K_{1}^{[2]} a_{\mathrm{A}}}
$$

Figure 5 shows that numerically simulated time-averaged rate for a system exhibiting resonance is in excellent agreement with the prediction made in eq. (22)—exemplifying the descriptive potence of allying simple, analytical mathematical methods with physical/chemical insight derived from algorithmic techniques for finding maximized dynamic catalytic rates. The familiarity of the Langmuir-Hinshelwood rate expression in eq. (22), for example, makes clear that, during dynamic catalytic resonance, (i) the ratecontrolling transition state is the conversion of $\mathrm{A}^{*} \rightarrow \mathrm{B}^{*}$ and (ii) the most abundant surface intermediate 
(MASI) in kinetic state 2 is determined by the activity of A (i.e. the MASI is bare sites, ${ }^{*}$, for $a_{\mathrm{A}} \ll K_{1}^{[2]}$ and $\mathrm{A}^{*}$ for $\left.a_{\mathrm{A}} \gg K_{1}^{[2]}\right)[32-34]$.

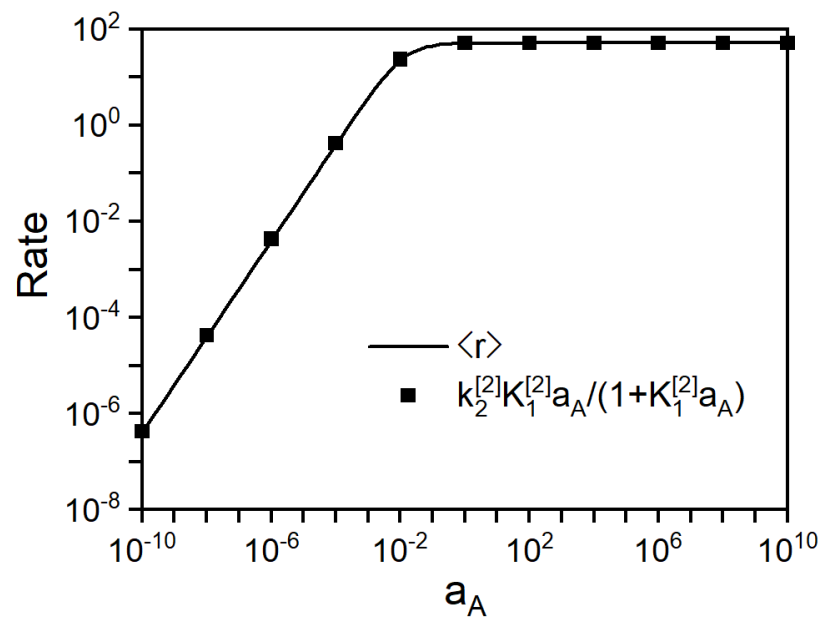

Figure 5. Time-averaged rate (solid line) compared to approximation in eq. (22) (square points) for $\mathrm{BE}_{\mathrm{B}}^{[1]}=$ $0 \mathrm{eV}, \mathrm{BE}_{\mathrm{B}}^{[2]}=1.03 \mathrm{eV}, \delta t^{[2]} / \delta t^{[1]}=10^{6}, a_{\mathrm{B}}=0, f=10^{3} \mathrm{~Hz}$, and $\log _{10}\left[k_{1}^{[1]}, k_{-1}^{[1]}, k_{2}^{[1]}, k_{-2}^{[1]}, k_{3}^{[1]}, k_{-3}^{[1]}\right]$ $=[6.00,31.91,10.07,-8.84,13.00,6.00]$.

With a closed-form approximation for the time-averaged rate during resonance in-hand, we next determine the necessary criteria and ideal operating conditions for dynamic catalysis resonance. For a system to exhibit resonance, kinetic state 2 (strong $\mathrm{BE}_{\mathrm{B}}$ ) must be more temporally efficient than kinetic state 1 (weak $\mathrm{BE}_{\mathrm{B}}$ ) at converting $\mathrm{A}$ to $\mathrm{B}^{*}$, otherwise kinetic state 1 would be better at executing all three elementary steps and forced kinetic oscillations would not increase the rate of reaction. This condition is formulated mathematically by requiring the derivative of eq. (22) with respect to $\mathrm{BE}_{\mathrm{B}}$ evaluated at $\mathrm{BE}_{\mathrm{B}}^{[1]}$ to be greater than zero (eq. (23)):

$$
\left.\frac{\mathrm{d}}{\mathrm{d} \mathrm{BE}_{\mathrm{B}}} \frac{k_{2} K_{1} a_{\mathrm{A}}}{1+K_{1} a_{\mathrm{A}}}\right|_{\mathrm{BE}_{\mathrm{B}}^{[1]}}=\frac{\left(1+K_{1}^{[1]} a_{\mathrm{A}}\right)\left(\omega_{-1}-\omega_{2}\right) k_{2}^{[1]} K_{1}^{[1]} a_{A}-k_{2}^{[1]} K_{1}^{[1]} a_{A}\left(\omega_{-1} K_{1}^{[1]} a_{\mathrm{A}}\right)}{\left(1+K_{1}^{[1]}\right)^{2} R T}>0
$$

After rearranging, eq. (23) simplifies to eq. (24): 


$$
\left(\omega_{-1}-\omega_{2}\right)>\omega_{-1} \frac{K_{1}^{[1]} a_{\mathrm{A}}}{1+K_{1}^{[1]} a_{\mathrm{A}}}
$$

In addition, for resonance to occur as described above, it is necessary for kinetic state 1 to regenerate a bare surface with little $\mathrm{A}^{*}$, i.e. $K_{1}^{[1]} a_{\mathrm{A}} \ll 1$, further simplifying the criteria to $\omega_{2}<\omega_{-1}$. Thus, the concerned resonance mechanism simply requires that the catalyst properties lie above the $\omega_{-1}=\omega_{2}$ iso-equilibrium line. The described mechanism of dynamic catalytic resonance does not, however, guarantee that $\langle r\rangle$ is greater than the Sabatier volcano maximum. In other words, while $\omega_{2}<\omega_{-1}$ ensures the resonance mechanism occurs per eq. (22), the degree of rate enhancement in reference to the Sabatier limit also depends on the relative magnitude of the rate constants, not only their scaling relationships. For example, if linear relationships hold indefinitely (i.e., $\Delta G_{j}^{\text {oł }}=\omega_{j} \mathrm{BE}_{\mathrm{B}}+b_{j}$ ), whether resonance occurs depends solely on the slopes $\omega_{j}$, but whether this resonance rate is larger than the Sabatier maximum reaction rate depends on the intercepts, $b_{j}$.

We aim to synthesize the prescriptions from eqs. (22)-(24) with learnings from analysis of quasistatic dynamic catalysis conditions (eqs. (20)-(21)) to assign to each pair $\left(\omega_{-1}, \omega_{2}\right)$ the mechanism of optimal rate enhancement via dynamic catalysis. The intersection of linear scaling correlations based on the mechanism of dynamic rate enhancement (quasi-static versus resonance) and optimal wave form (symmetric versus asymmetric) forms a landscape of distinct catalyst types which we report in Figure 6. Each region of Figure 6 is bound by derived linear scaling correlations (e.g., iso-equilibrium lines $\omega_{-1}=$ $\omega_{2}$ and $\left.\omega_{-1}=1\right)$ and is categorized by the optimal method for achieving enhanced rates or dynamic equilibrium conversion: resonance, quasi-static symmetric oscillation, or quasi-static asymmetric oscillation. Catalysts are further classified into twelve different sub-types based on the correlations between elementary step free energies of activation with the activation free energy for product desorption, i.e., the signs of $\omega_{-1}, \omega_{2}$ and $\omega_{1}=1+\omega_{2}-\omega_{-1}$. 
In Figure 6, we delineate the catalyst types for all possible $\omega_{j}$ without making any distinction on the physical bounds of these catalyst properties. Previous work $[18,27]$ on the reaction sequence in Scheme 3 has expressed the linear scaling relations in terms of $\gamma \equiv \Delta \mathrm{BE}_{\mathrm{B}} / \Delta \mathrm{BE}_{\mathrm{A}}$, which can take any value, and $\alpha \equiv \Delta E_{\mathrm{a}, \mathrm{sr}} / \Delta H_{\mathrm{sr}}$, which relates the activation energy of the surface reaction to the enthalpy change of the surface reaction. Precedent literature [31] suggests $\alpha$ is typically bounded from 0 to 1 , though exceptions likely exist. The $\omega_{j}$ are related to $\alpha$ and $\gamma$ by $\omega_{-1}=1 / \gamma$ and $\omega_{2}=\alpha(1 / \gamma-1)$, and thus only catalyst types II, VI, X, and XI fall in the range of $0 \leq \alpha \leq 1$. The iso-equilibrium lines in terms of $\alpha$ and $\gamma$ become $\gamma=1$ and $\alpha=1 /(1-\gamma)$. One point on the latter iso-equilibrium line $(\gamma=-1.5, \alpha=0.4)$ was discovered by Gathmann et al. [35] to achieve supra-equilibrium conversion at quasi-static conditions, the inequality $\alpha>1 /(1-\gamma)$ must be satisfied. A variable transformation of Figure 6 in terms of $\alpha$ and $\gamma$ is reported in Section S2 of the Supporting Information.

Catalyst types V, VI, IX, and X are labeled as quasi-static dynamic catalysts in Figure 6, but also all exhibit dynamic catalysis "resonance." However, there always exists a quasi-static dynamic catalysis condition that yields higher rates and conversions. If there are physical limitations on amplitude and frequencies of kinetic oscillations, then operating these catalyst types as resonance catalysts may be preferable. Likewise, catalyst type VII is both a resonance and asymmetric quasi-static dynamic catalyst and is colored as such to distinguish it from catalyst type IV, which does not exhibit any resonance regimes. Theoretically, the asymmetric quasi-static catalysts achieve the same rates and conversions as symmetric quasi-static dynamic catalysts; however, the asymmetry of the oscillation waves may require one kinetic 
state to persist for sub-femtosecond timescales (see eq. (14)), making resonance conditions more practical for type VII catalysts.

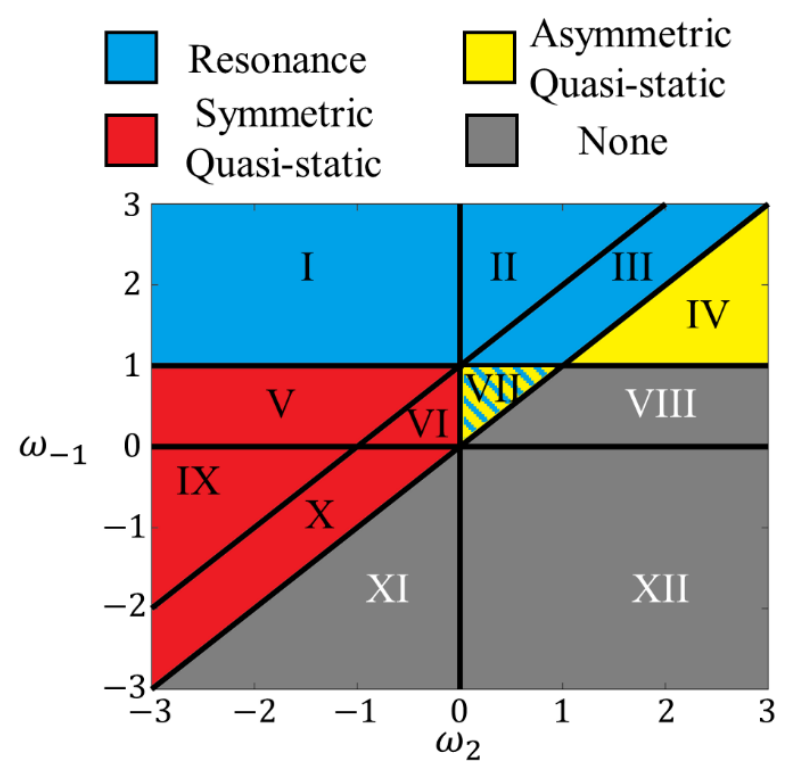

Figure 6. The dynamic catalysis behavior as a function of $\omega_{2}$ and $\omega_{-1}$. Catalyst types are further subcategorized into twelve sub-types based on the correlation of activation energies of elementary steps with the activation energy of product desorption. Enhanced rates and supra-equilibrium conversion are achieved: (i) in the blue region (types I-III and VII) by oscillating at a range of resonance frequencies, (ii) the yellow region (types IV and VII) by operating with an asymmetric square wave at quasi-static surface frequencies, and (iii) the red region (types V, VI, IX, and X) by symmetric or asymmetric square-wave oscillation at quasi-static surface frequencies. In the gray region (types VIII, XI, and XII), little to no rate or equilibrium enhancement is achieved by square-wave oscillations at quasi-static surface conditions for $A+* \rightarrow B^{*}$ type resonance frequencies. Other resonance types $\left(A^{*} \rightarrow B+*\right.$ or $\left.A+B^{*} \rightarrow B+A^{*}\right)$ may occur for these catalyst types but these are beyond the scope of the present work.

Figure 6 details the categorization of catalysts based on their dynamic catalysis properties and further sub-categorized into twelve different types based on free energy correlations. Catalysts in the same sub-type (I-XII) will behave nearly identically during dynamic catalysis, while catalysts with the same dynamic catalysis properties (indicated by colors in Figure 6) will exhibit similar behavior. Thus, we will not detail an example for each of the twelve sub-types, but instead we next highlight general behaviors in select instructive case studies. 


\subsubsection{Volcano plots for dynamic catalysis}

The first case study we consider is resonance for a catalyst of sub-type III with $\omega_{2}=1$ and $\omega_{-1}=$ 1.5. We develop a two-dimensional volcano plot by systematically varying $\mathrm{BE}_{\mathrm{B}}^{[1]}$ and $\mathrm{BE}_{\mathrm{B}}^{[2]}$ and then evaluating the rate at three frequencies: below both eigenvalues for kinetic state 2 (steady-state kinetics), a geometric average of both eigenvalues (in the range of resonance frequencies if it occurs), and above both eigenvalues (quasi-static surface kinetics). The optimal reaction rate for each $\mathrm{BE}_{\mathrm{B}}^{[j]}$ combination is shown away from thermodynamic equilibrium (Figure 7a) and at thermodynamic equilibrium (Figure 7b). In Figure $7 \mathrm{a}$ and Figure $7 \mathrm{~b}$, the diagonal $\mathrm{BE}_{\mathrm{B}}^{[1]}=\mathrm{BE}_{\mathrm{B}}^{[2]}$ corresponds to the static catalysis volcano plot. The dynamic rate is symmetric about $\mathrm{BE}_{\mathrm{B}}^{[1]}=\mathrm{BE}_{\mathrm{B}}^{[2]}$ because $\left(\mathrm{BE}_{\mathrm{B}}^{[1]}, \mathrm{BE}_{\mathrm{B}}^{[2]}\right)=(x, y)$ and $\left(\mathrm{BE}_{\mathrm{B}}^{[1]}, \mathrm{BE}_{\mathrm{B}}^{[2]}\right)=(y, x)$ are identical oscillations shifted temporally, and thus give the same reaction rate. The optimal resonance frequencies are weak functions of $a_{\mathrm{B}}$, as shown by comparison of Figure $7 \mathrm{c}$ to Figure $7 \mathrm{~d}$.

For $\omega_{2}=1, \omega_{-1}=1.5$, the catalyst does not exhibit enhanced reaction rates at high oscillation frequency (Figure 4) but does exhibit enhanced reaction rates at resonance frequencies (Figure 7). At this resonance condition, the weaker binding energy of $\mathrm{B}^{*}$ needs to be sufficiently small, and the rate is highest when the stronger binding energy is a specific value; for the conditions shown in Figure 7, this value is $\mathrm{BE}_{\mathrm{B}}^{[2]} \sim 0.7 \mathrm{eV}$. We showed in Figure 5 that the time-averaged reaction rate is approximated by the rate of the overall reaction $\mathrm{A}+* \rightarrow \mathrm{B}^{*}$ (eq. (22)). The rate of $\mathrm{A}+* \rightarrow \mathrm{B}^{*}$ (eq. (22)) is shown as a function of $\mathrm{BE}_{\mathrm{B}}^{[2]}$ in Figure 8 to illustrate that the rate of this reaction is described by a volcano plot whose peak is located at $\sim 0.7 \mathrm{eV}$, the same optimal $\mathrm{BE}_{\mathrm{B}}^{[2]}$ for resonance in Figure 7 .

While the overall reaction involves three reaction steps $\left(A+* \rightarrow A^{*}, A^{*} \rightarrow B^{*}, B^{*} \rightarrow B+*\right)$, resonance effectively simplifies the kinetically-relevant reaction path to the two steps executed during kinetic state $2\left(\mathrm{~A}+* \rightarrow \mathrm{A}^{*}\right.$ and $\left.\mathrm{A}^{*} \rightarrow \mathrm{B}^{*}\right)$, with the desorption of $\mathrm{B}^{*}$ as a kinetically-irrelevant step. The $\mathrm{BE}_{\mathrm{B}}^{[2]}$ needs to be optimized with respect to the sub-reaction $\mathrm{A}+* \rightarrow \mathrm{B}^{*}$, as illustrated by the volcano plot 
in Figure 8a. Thus, for dynamic catalysis resonance, the reaction rate is still constrained by the Sabatier principle for static catalysis, but for a subset of the overall reaction network.

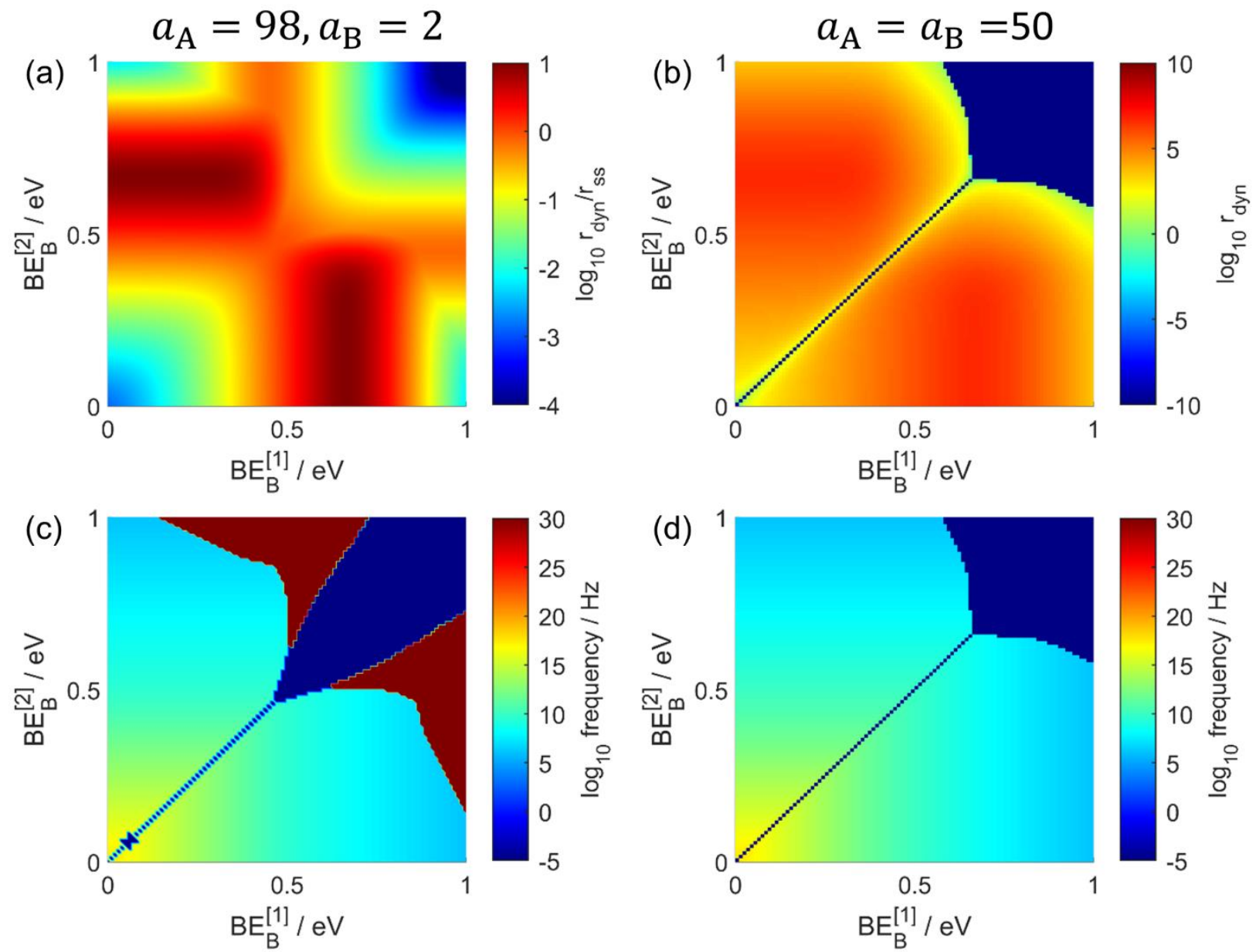

Figure 7. (a) A projection of the multi-dimensional dynamic catalysis volcano plot for a square wave oscillation with endpoints $\mathrm{BE}_{\mathrm{B}}^{[1]}$ and $\mathrm{BE}_{\mathrm{B}}^{[2]}$ operating at an optimal frequency at $a_{\mathrm{A}}=98, a_{\mathrm{B}}=2$ and (b) at thermodynamic equilibrium $\left(a_{\mathrm{A}}=a_{\mathrm{B}}=50\right)$. (c) The optimal frequency as a function of $\mathrm{BE}_{\mathrm{B}}^{[1]}$ and $\mathrm{BE}_{\mathrm{B}}^{[2]}$ at $a_{\mathrm{A}}=98, a_{\mathrm{B}}=2$ and (d) thermodynamic equilibrium $a_{\mathrm{A}}=a_{\mathrm{B}}=50$. For (a)-(d), Type III catalyst with $\omega_{2}=1, \omega_{-1}=1.5, \quad$ and $\quad \mathrm{T}=373 \mathrm{~K}$. At $\quad \mathrm{BE}_{\mathrm{B}}=0 \mathrm{eV}, \quad \log _{10}\left[k_{1}, k_{-1}, k_{2}, k_{-2}, k_{3}, k_{-3}\right]=$ $[6.00,22.45,17.63,8.18,13.00,6.00]$. Dark red regions in the frequency plots correspond to quasi-static conditions, dark blue corresponds to the limit as $f \rightarrow 0$ (time-average of static steady state rates), and intermediate regions are "resonant" frequencies. 

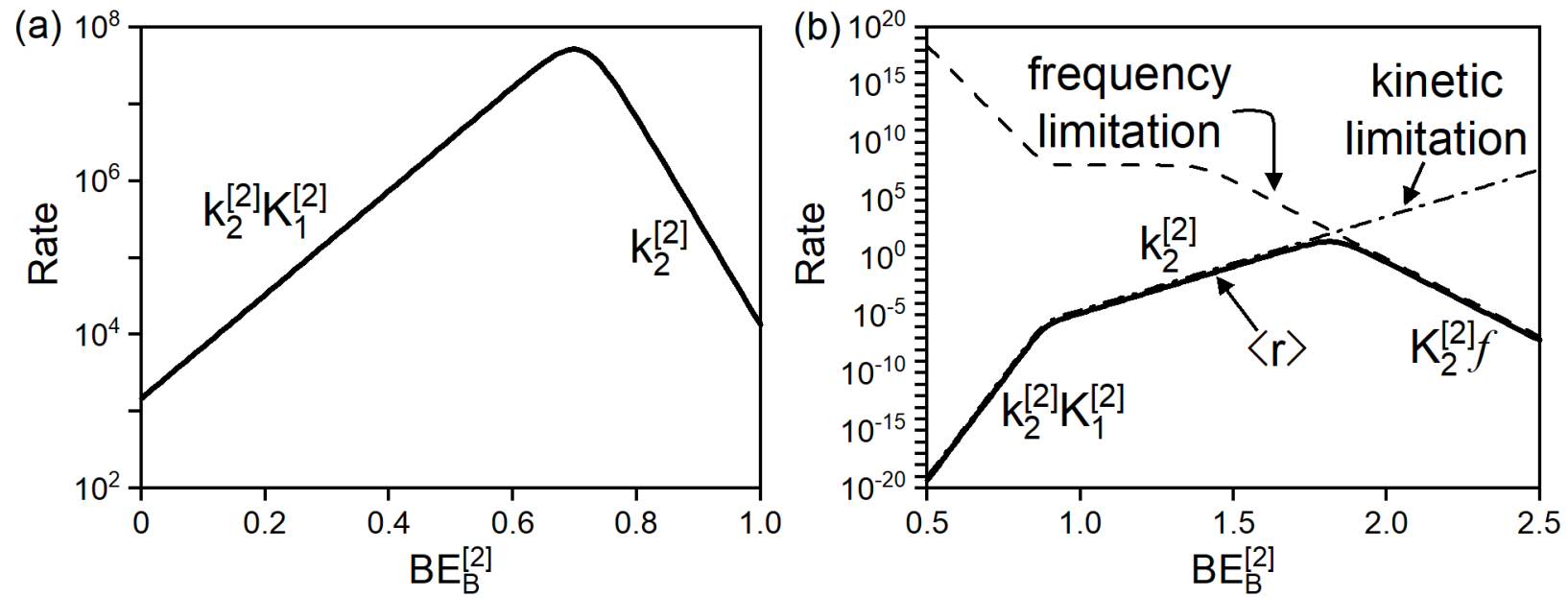

Figure 8. (a) The volcano plot for resonance behavior for Type II, III, and VII catalysts in Figure 6. Shown here is a Type II catalyst with $a_{\mathrm{A}}=100, a_{\mathrm{B}}=0, \delta=1.4 \mathrm{eV}, \mathrm{T}=373 \mathrm{~K}, \omega_{2}=1, \omega_{-1}=1.5$. (b) The volcano plot for resonance behavior of Type I catalysts in Figure 6. The specific properties are $\omega_{2}=-0.6$, $\omega_{-1}=2$. For both (a) and (b), $a_{\mathrm{A}}=100, a_{\mathrm{B}}=0, \quad \delta t^{[2]} / \delta t^{[1]}=100, \quad \mathrm{BE}_{\mathrm{B}}^{[1]}=0 \mathrm{eV}$, $\log _{10}\left[k_{1}^{[1]}, k_{-1}^{[1]}, k_{2}^{[1]}, k_{-2}^{[1]}, k_{3}^{[1]}, k_{-3}^{[1]}\right]=[6.00,31.91,10.07,-8.84,13.00,6.00]$. The frequency was chosen as the center of the resonance region, $f=\sqrt{\lambda_{1}^{[2]} \lambda_{2}^{[2]}}$.

Figure $7 \mathrm{~b}$ shows that the rate is positive for dynamic systems in resonance even when the reactant and products are at thermodynamic equilibrium. In eq. (21), $K_{\mathrm{dyn}}$ is an exponential function of the oscillation amplitude, $\Delta \mathrm{BE}_{\mathrm{B}}^{[2]}$, at quasi-static conditions, but eq. (21) does not apply at resonance frequencies. Determination of $K_{\mathrm{dyn}}$ for dynamic catalysis resonance requires a different approach. We derive an expression for $K_{\mathrm{dyn}}$ at resonance conditions by first recognizing that the dynamic equilibrium is defined as the condition where the dynamic reaction rate is zero. Figure 5 illustrates that, in the absence of product, eq. (22) describes well the time-averaged reaction rate for the resonance mechanism of dynamic operation of the reaction in Scheme 3. Deriving a general expression for the dynamic reaction rate, however, is intractable without making any simplifying assumptions; and, the nature of the requisite approximations required to describe the rate during dynamic resonance in the presence of product is not immediately clear. To find a reasonable approximation to the minimum $K_{\mathrm{dyn}}$, we consider the following thought experiment putatively re-enacting the mechanism of resonance in the presence of B: (i) during kinetic state 1, a bare surface is generated by desorbing all $\mathrm{A}^{*}$ to $\mathrm{A}$ and all $\mathrm{B} *$ to $\mathrm{B}$; (ii) kinetic state 2 persists for a short time 
such that the surface remains mostly bare $\left(\theta_{*}(t) \approx 1\right)$. At these short timescales, we posit that $\theta_{\mathrm{A}^{*}} \approx$ $k_{1}^{[2]} a_{\mathrm{A}} t$ and $\theta_{\mathrm{B}^{*}} \approx k_{-3}^{[2]} a_{\mathrm{B}} t$ and the surface reaction step is rate-determining such that the time-averaged rate of the limit cycle is proportional to the net rate of $A^{*} \rightarrow B^{*}$ (eq. (25)):

$$
r_{\mathrm{A}^{*} \rightarrow \mathrm{B}^{*}}(t)=k_{2}^{[2]} \theta_{\mathrm{A}^{*}}(t)-k_{-2}^{[2]} \theta_{\mathrm{B}^{*}}(t)=\left(k_{1}^{[2]} k_{2}^{[2]} a_{\mathrm{A}}-k_{-2}^{[2]} k_{-3}^{[2]} a_{\mathrm{B}}\right) t
$$

Thus, at worst, the reaction is driven in the forward direction if eq. (25) is positive, which sets an absolute lower bound on the optimal $K_{\text {dyn }}$ (eq. (26)):

$$
K_{\mathrm{dyn}}=\frac{a_{\mathrm{B}}^{\mathrm{eq}}}{a_{\mathrm{A}}^{\mathrm{eq}}}=\frac{k_{1}^{[2]} k_{2}^{[2]}}{k_{-2}^{[2]} k_{-3}^{[2]}}=K_{2}^{[2]}
$$

since $k_{1}^{[2]}=k_{-3}^{[2]}$ in this example. We find that eq. (26) is not just a lower bound, but also an excellent approximation of the dynamic equilibrium constant, as shown in Figure 9, where the time-averaged rate is zero when $a_{\mathrm{B}} / a_{\mathrm{A}}=K_{2}^{[2]}$ for a catalyst exhibiting resonance. This relationship is explicated more rigorously by deriving a closed-form solution for the rate in the presence of product by reducing the complexity of the model. This is accomplished by assuming that the desorption of $\mathrm{B}^{*}$ and the interconversion of $\mathrm{A}^{*}$ and $\mathrm{B}^{*}$ do not impact the dynamics of the system (i.e., the eigenvalues), but do alter the reaction rate. Further, the optimal time $\delta t^{[2]}$ is assumed equal to $\sqrt{\lambda_{1}^{[2]} \lambda_{2}^{[2]}}$, which the center of the resonance frequency range. The resulting solution for the net rate of $\mathrm{A}^{*}$ to $\mathrm{B}^{*}$ formation during resonance was found by using the symbolic solver in Matlab (eq. (27):

$$
\langle r\rangle \approx\left(k_{1}^{[2]} k_{2}^{[2]} a_{\mathrm{A}}-k_{-2}^{[2]} k_{-3}^{[2]} a_{\mathrm{B}}\right) \chi
$$




$$
\begin{gathered}
\chi=\frac{2 \sqrt{k_{-1}^{[2]} k_{-3}^{[2]} a_{\mathrm{B}}}}{\left(1+\delta t^{[1]} / \delta t^{[2]}\right)\left(k_{1}^{[2]} a_{\mathrm{A}}+k_{-1}^{[2]}+k_{-3}^{[2]} a_{\mathrm{B}}-\Lambda\right) \Lambda} \\
\times\left(1-\exp \left(-\frac{k_{1}^{[2]} a_{\mathrm{A}}+k_{-1}^{[2]}+k_{-3}^{[2]} a_{\mathrm{B}}-\Lambda}{2 \sqrt{k_{-1}^{[2]} k_{-3}^{[2]} a_{\mathrm{B}}}}\right)\right) \\
\Lambda=\left[\left(k_{1}^{[2]} a_{\mathrm{A}}\right)^{2}+\left(k_{-3}^{[2]} a_{\mathrm{B}}\right)^{2}+\left(k_{-1}^{[2]}\right)^{2}+2\left(k_{1}^{[2]} k_{-1}^{[2]} a_{\mathrm{A}}+k_{1}^{[2]} k_{-3}^{[2]} a_{\mathrm{B}}-k_{-1}^{[2]} k_{-3}^{[2]} a_{\mathrm{B}}\right)\right]^{1 / 2}
\end{gathered}
$$

Equation (27) is in excellent agreement with the numerically simulated reaction rate, as shown in Figure 9. Following a similar procedure, the optimal asymmetry ratio $\delta t^{[1]} / \delta t^{[2]}$ can also be written in explicit terms of rate constants to obtain a closed-form solution which we omit for the sake of brevity. Even with the model simplification, eq. (27) is quite complex but, nevertheless, evidently and unequivocally confirms that (i) at $a_{\mathrm{B}} / a_{\mathrm{A}}=k_{1}^{[2]} k_{2}^{[2]} / k_{-2}^{[2]} k_{-3}^{[2]}=K_{2}^{[2]}$, the net rate is zero, in agreement with our thought experiment detailed above (eq. (25)) and (ii) for dynamic catalysis resonance, the dynamic equilibrium constant is not a function of the oscillation amplitude and does not depend on the thermodynamic equilibrium constant - further illustrating the fundamental differences between quasi-static and resonance conditions. Furthermore, eq. (27) demonstrates that there is no guarantee that $K_{2}^{[2]}$ will be greater than $K$ of the overall reaction, indicating that not all systems with the appropriate $\omega_{j}$ values will exhibit resonant equilibrium enhancement. Just as the case for resonant rate enhancement, whether a resonant dynamic catalyst drives reactions against a thermodynamic gradient depends not only on the slopes of the scaling relations, $\omega_{j}$, but also on their intercepts (i.e., not all catalysts above the $\omega_{2}=\omega_{-1}$ line are resonance catalysts). A final feature of resonance we highlight is the significant role of product inhibition; as B and A both rapidly adsorb on the catalyst during kinetic state $2, \mathrm{~B}^{*}$ effectively blocks the adsorption of $\mathrm{A}$, reducing both the reaction rate and temporal efficiency of dynamic forcing. 


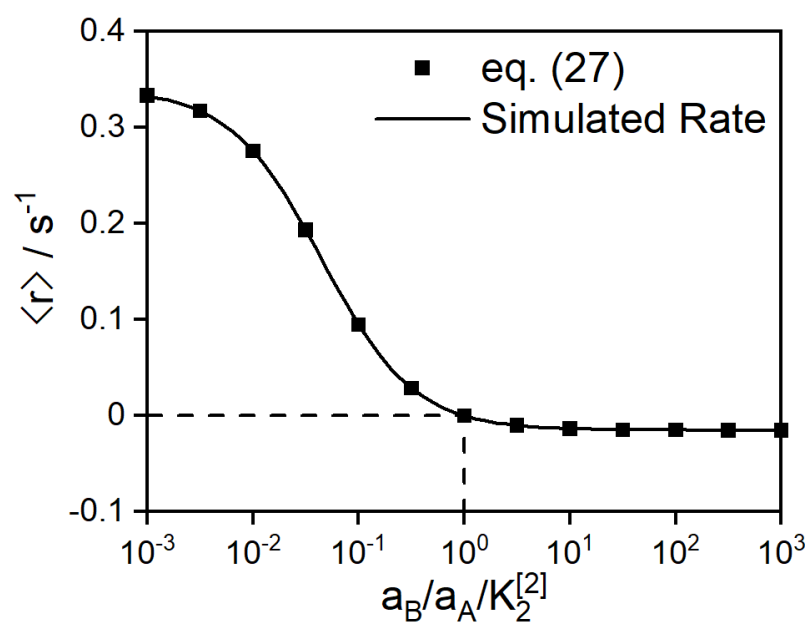

Figure 9. Rate as a function of $a_{\mathrm{B}} / a_{\mathrm{A}} / K_{2}^{[2]}$. The rate is zero at $a_{\mathrm{B}} / a_{\mathrm{A}} / K_{2}^{[2]}=1$ because $K_{\mathrm{dyn}} \approx K_{2}^{[2]}$ in agreement with eq. (25) A frequency at the center of the resonance region was chosen as $f=\sqrt{\lambda_{1}^{[2]} \lambda_{2}^{[2]}}$. The square points are an analytical approximation (eq. (27)) and the line is a numerical simulation. Conditions: $\quad \omega_{2}=0.6, \omega_{-1}=2, \mathrm{BE}_{\mathrm{B}}^{[1]}=0 \mathrm{eV}, \mathrm{BE}_{\mathrm{B}}^{[2]}=1.3 \mathrm{eV}, \quad a_{A}=50$, $\log _{10}\left[k_{1}^{[1]}, k_{-1}^{[1]}, k_{2}^{[1]}, k_{-2}^{[1]}, k_{3}^{[1]}, k_{-3}^{[1]}\right]=[6.00,31.91,10.07,-8.84,13.00,6.00] . K_{2}^{[2]}=22.42$ and $a_{\mathrm{B}}$ was varied.

The behavior for a type II catalyst shown in Figure 7 and Figure $8 \mathrm{a}$ is generally representative of the dynamic catalysts exhibiting resonance (types I-III and VII in Figure 6), with one exception. For catalyst types II, III, and VII, eq. (22) has a maximum located at $\mathrm{BE}_{\mathrm{B}}^{[2]}$ where $K_{1}^{[2]} \sim 1$ that is designated as the volcano peak (Figure 8a). However, for type I catalysts $\left(\omega_{2}<0, \omega_{-1}>1\right)$, eq. (22) increases without bound because $k_{2}^{[2]} \propto \exp \left(-\omega_{2} \mathrm{BE}_{\mathrm{B}}^{[2]} / R T\right)$ and $K_{1}^{[2]} \propto \exp \left(\omega_{-1} \mathrm{BE}_{\mathrm{B}}^{[2]} / R T\right)$ both diverge to infinity with increasing $\mathrm{BE}_{\mathrm{B}}^{[2]}$. This would suggest that the dynamic catalysis rate would increase indefinitely with increasing $\mathrm{BE}_{\mathrm{B}}^{[2]}$ at resonance conditions, but this is not observed (Figure 8b). Instead, at binding energies $\mathrm{BE}_{\mathrm{B}}^{[2]}$ much higher than where $K_{1}^{[2]} \sim 1$, a maximum rate is reached and the rate decreases with increasing $\mathrm{BE}_{\mathrm{B}}^{[2]}$ thereafter. We identify this peak as the point where there is a regime change in the rate-limiting process, transitioning from kinetic limitation to oscillation frequency limitation. 
In the oscillation-frequency-limited regime (i.e., high $\mathrm{BE}_{\mathrm{B}}^{[2]}$ ), $\mathrm{B}^{*}$ rapidly equilibrates with $\mathrm{A}^{*}$ on the surface, and thus the fractional coverage of $\mathrm{B}^{*}$ during kinetic state 2 is (eq. (28)):

$$
\theta_{\mathrm{B}^{*}}^{[2]} \approx \frac{K_{1}^{[2]} K_{2}^{[2]} a_{\mathrm{A}}}{1+K_{1}^{[2]} a_{\mathrm{A}}+K_{1}^{[2]} K_{2}^{[2]} a_{\mathrm{A}}} \approx K_{2}^{[2]}
$$

where $K_{1}^{[2]} a_{\mathrm{A}} \gg 1, K_{1}^{[2]} K_{2}^{[2]} a_{\mathrm{A}}$ (i.e., $\theta_{\mathrm{A}^{*}}^{[2]} \approx 1$ ). In kinetic state $1, \mathrm{~B}^{*}$ is removed from the surface and converted to $\mathrm{B}$, with a maximum yield of $\theta_{\mathrm{B}^{*}}^{[2]}$. Thus, the rate of this reaction is equal to $\theta_{\mathrm{B}^{*}}^{[2]}$ multiplied by the number of oscillations per unit time, or the frequency, $f$. This frequency cannot be infinitely fast because it needs to remain in the resonance regime (otherwise the reaction will enter the quasi-static regime which does not exhibit rate enhancement for type I catalysts, as was illustrated in Figure 4), and thus this limit on the oscillation frequency imposes an upper bound on the reaction rate. The rate-control of the oscillationfrequency limit for sufficiently large $\mathrm{BE}_{\mathrm{B}}^{[2]}$ is evident in Figure $8 \mathrm{~b}$ which illustrates that the kinetic bound per eq. (22), shown by the dash-dot line, and $\theta_{\mathrm{B}^{*}}^{[2]} f$ per eq. (28), shown by the dashed line, respectively coincide with $\langle r\rangle$ as a function of $\mathrm{BE}_{\mathrm{B}}^{[2]}$. Therefore, at low $\mathrm{BE}_{\mathrm{B}}^{[2]}$, the reaction is kinetically limited by the conversion of $\mathrm{A}^{*} \rightarrow \mathrm{B}^{*}$, and at high $\mathrm{BE}_{\mathrm{B}}^{[2]}$ the reaction is limited by the maximum resonance frequency. The optimal $\mathrm{BE}_{\mathrm{B}}^{[2]}$ sits at the transition between these two kinetic regimes and is approximately where $k_{2}^{[2]}=$ $K_{2}^{[2]} f$ which simplifies to $f=k_{-2}^{[2]}$. Thus, resonance in type I catalysts also exhibits volcano-plot-like behavior, except instead of each regime corresponding exclusively to different kinetic or thermodynamic limitations, the turnover frequency for these catalysts can be limited by the frequency of the kinetic oscillation. 
We showed above that resonance catalysts have the property that $\omega_{-1}>\omega_{2}$, which includes catalyst types I-III and VII, but also types V, VI, IX, and X. When operating at frequencies in the resonance regime, catalyst types V, VI, IX, and X will exhibit similar behavior to that shown in Figure 7-Figure 9. However, these four catalyst types are best operated at the high frequency limit under quasi-static conditions. Catalysts of these types have multi-variable volcano plots like those shown in Figure 10a. The static kinetics volcano plot has a Sabatier maximum at $\mathrm{BE}_{\mathrm{B}}^{[1]}=\mathrm{BE}_{\mathrm{B}}^{[2]} \sim 1.5 \mathrm{eV}$; the dynamic kinetics volcano plateaus near $\mathrm{BE}_{\mathrm{B}}^{[1]}<0.5 \mathrm{eV}, \mathrm{BE}_{\mathrm{B}}^{[2]}>2.0 \mathrm{eV}$, where further decreasing $\mathrm{BE}_{\mathrm{B}}^{[1]}$ or increasing $\mathrm{BE}_{\mathrm{B}}^{[2]}$ has negligible impact on the time-averaged rate. At these conditions, the reaction rate is $\langle r\rangle \approx k_{1} a_{\mathrm{A}}=$ $9.8 \times 10^{7} \mathrm{~s}^{-1}$, implying that the adsorption of $\mathrm{A}$ is the rate-controlling process for the conversion of $\mathrm{A}$ to $\mathrm{B}$ at these conditions. The rate constant $k_{1}$ is not a function of $\mathrm{BE}_{\mathrm{B}}$, and the rate of this step cannot be increased. At these conditions, the Sabatier limit has been completely circumvented; the rate of conversion is no longer limited by the fundamental kinetics of the reaction, but instead is limited by the rate at which reactant $\mathrm{A}$ adsorbs on the catalyst surface.

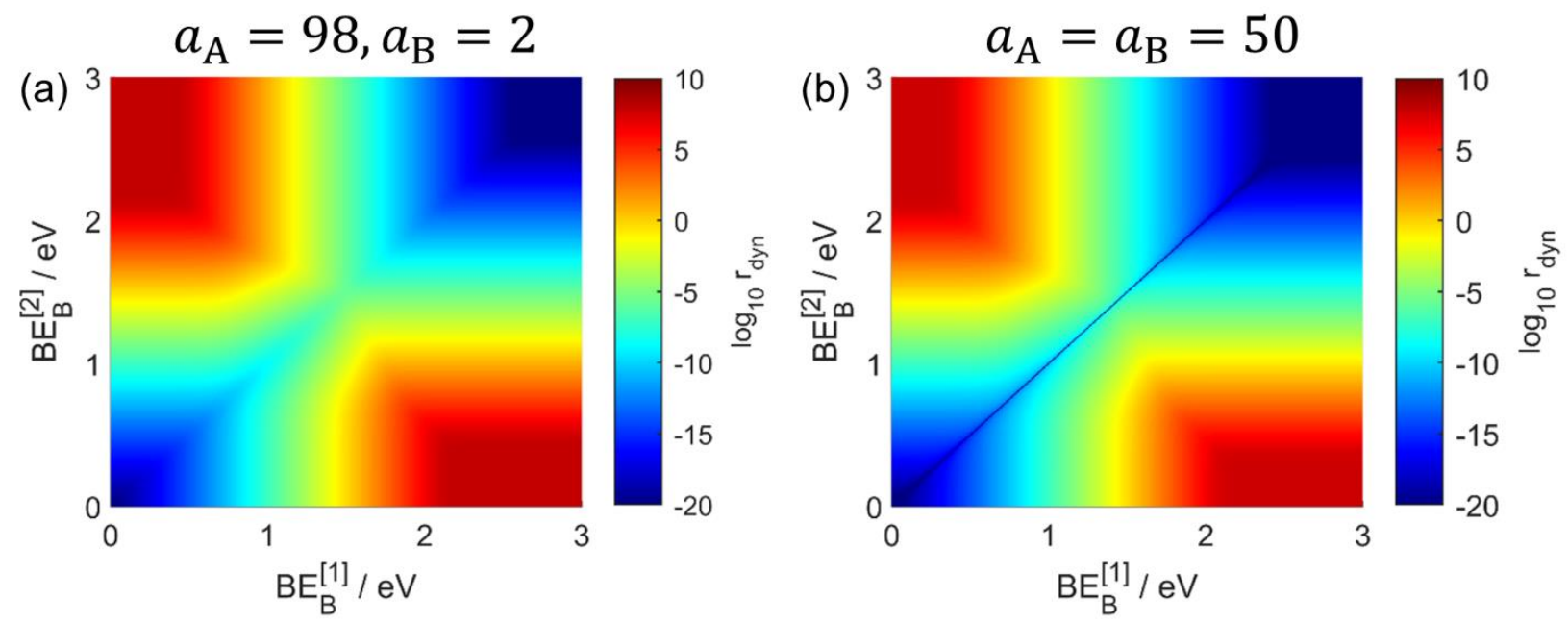

Figure 10. The multi-dimensional dynamic catalysis volcano plot at quasi-static conditions with $\omega_{2}=$ $-1, \omega_{-1}=-0.5$ At $\quad \mathrm{BE}_{\mathrm{B}}=0 \mathrm{eV}, \quad \log _{10}\left[k_{1}, k_{-1}, k_{2}, k_{-2}, k_{3}, k_{-3}\right]=$ $[6.00,-15.36,-20.19,8.18,13.00,6.00]$ (a) $a_{\mathrm{A}}=98, a_{\mathrm{B}}=2$ and (b) thermodynamic equilibrium $\left(a_{\mathrm{A}}=\right.$ $a_{\mathrm{B}}=50$ ). The diagonals in (a) and (b) are the static catalysis volcano plots, which is 0 at all points at thermodynamic equilibrium in (b). 
Recall from the analysis of $K_{\mathrm{dyn}}$ at quasi-static conditions (eq. (21)) that the dynamic equilibrium limit was solely a function of $\Delta \mathrm{BE}_{\mathrm{B}}=\mathrm{BE}_{\mathrm{B}}^{[2]}-\mathrm{BE}_{\mathrm{B}}^{[1]}$ and not of the values of the oscillation endpoints themselves. While $K_{\mathrm{dyn}}$ is only a function of the amplitude, the reaction rate at thermodynamic equilibrium $\left(a_{\mathrm{A}}=a_{\mathrm{B}}\right)$ is a function of the oscillation endpoints, as shown in Figure 10b. In Figure 10b, the rate is positive everywhere on the dynamic volcano plot except at static conditions $\left(\mathrm{BE}_{\mathrm{B}}^{[2]}=\mathrm{BE}_{\mathrm{B}}^{[1]}\right)$ where the rate is zero. Aside from the $\mathrm{BE}_{\mathrm{B}}^{[2]}=\mathrm{BE}_{\mathrm{B}}^{[1]}$ line, the rate does not perceptibly change between nearly irreversible (Figure 10a) and thermodynamic equilibrium (Figure 10b) conditions. This is because at static catalysis conditions, the reaction rate of a reversible reaction is given by $\langle r\rangle=\langle\vec{r}\rangle\left(1-a_{\mathrm{B}} / a_{\mathrm{A}} / K_{\mathrm{dyn}}\right)$, where $\langle\vec{r}\rangle$ is the time-averaged forward reaction rate. For the catalyst in Figure 10a, there is no product inhibition so $\langle\vec{r}\rangle$ is not a function of $a_{\mathrm{B}}$, and because $K_{\mathrm{dyn}} \gg K$ for even modest oscillation amplitudes due to its exponential dependence on $\triangle \mathrm{BE}_{\mathrm{B}}$, the dynamic kinetic rates are nearly unimpeded by the accumulation of product at thermodynamic equilibrium (Figure 10b).

The final case study we consider is that of a type IV catalyst that exhibits enhanced dynamic equilibrium under asymmetric kinetic oscillations. In Figure 11, the reaction rate and optimal asymmetry at irreversible ((a) and (b)) and thermodynamic equilibrium ((c) and (d)) conditions are reported. At irreversible conditions, the rate is only increased by $\sim 2 \times$ over the Sabatier maximum by asymmetric oscillations, suggesting that this catalyst type is not optimal for increasing reaction rates. However, at thermodynamic equilibrium (Figure $11 \mathrm{~b}$ ), the reaction rate is positive at all $\mathrm{BE}_{\mathrm{B}}^{[2]} \neq \mathrm{BE}_{\mathrm{B}}^{[1]}$, and the dynamic equilibrium constant is theoretically driven to infinity with large oscillation amplitudes by these asymmetric oscillations (eq. (21)), and thus these catalysts are good for driving reactions beyond thermodynamic limits. One challenge for these catalysts is that the optimal ratio $\delta t^{[2]} / \delta t^{[1]}$ for maximizing dynamic equilibrium is exponentially dependent on the oscillation amplitude (eq. (14)), as is $K_{\text {dyn }}$ (eq. (21)), and thus achieving large $K_{\text {dyn }}$ with type IV catalysts requires large $\delta t^{[2]} / \delta t^{[1]}$; when compounded with the high frequency of the oscillation, this may be technically infeasible in real applications. 

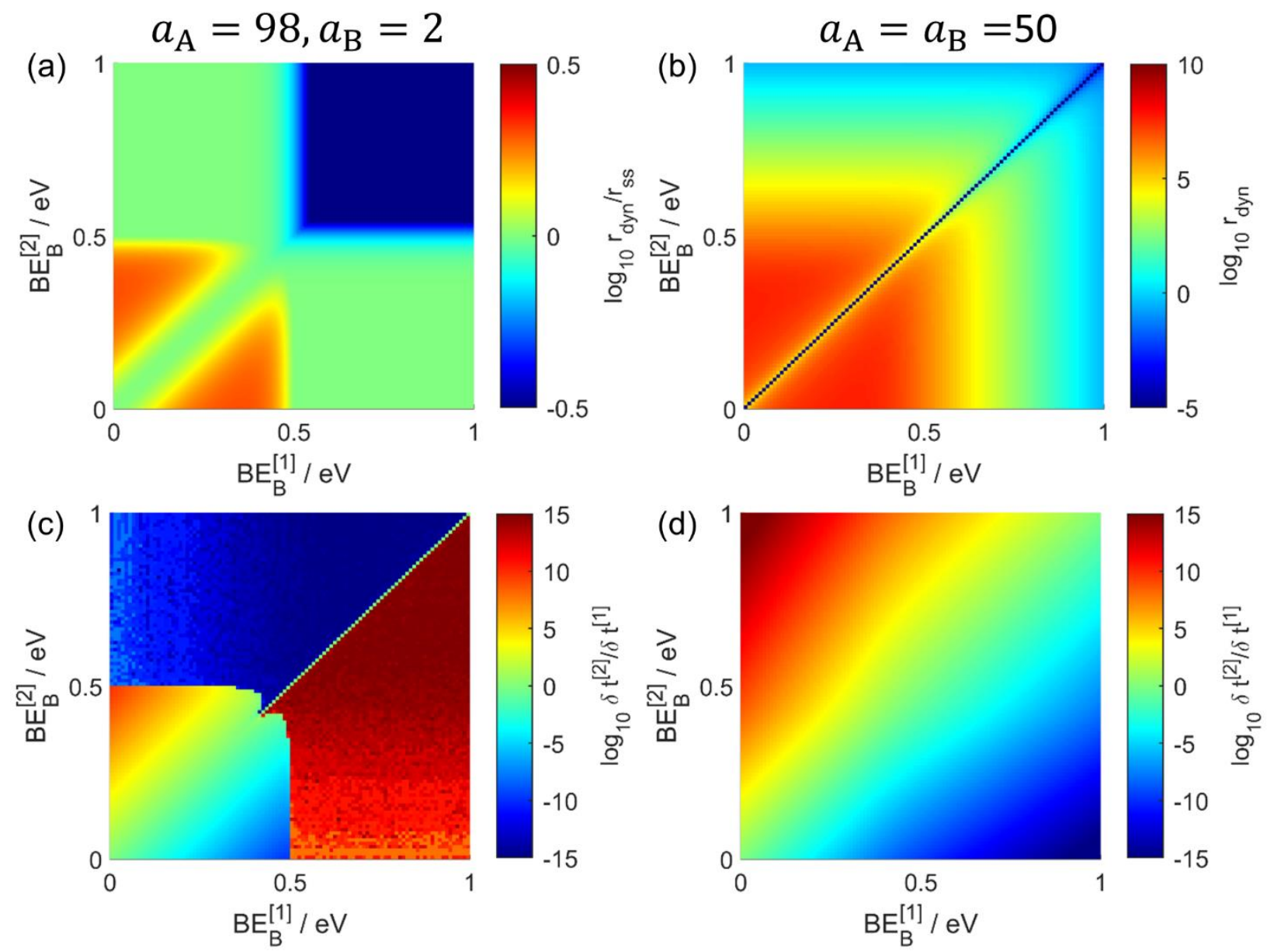

Figure 11. Multi-dimensional dynamic catalysis volcano plots for asymmetric oscillations for a catalyst with $\omega_{2}=2, \omega_{-1}=1.5$ and (a) $a_{\mathrm{A}}=98, a_{\mathrm{B}}=2$ or (b) $a_{\mathrm{A}}=a_{\mathrm{B}}=50$. The optimal $\delta t^{[2]} / \delta t^{[1]}$ ratio for (c) $a_{\mathrm{A}}=98, a_{\mathrm{B}}=2$ and (d) $a_{\mathrm{A}}=a_{\mathrm{B}}=50$. For both simulations, at $\mathrm{BE}_{\mathrm{B}}=0 \mathrm{eV}$, $\log _{10}\left[k_{1}, k_{-1}, k_{2}, k_{-2}, k_{3}, k_{-3}\right]=[6.00,22.45,36.54,27.08,13.00,6.00]$.

\subsubsection{Identifying dynamic catalyst properties for enhanced selectivity and yield}

Heretofore, we have detailed a methodology for identifying catalyst properties necessary to achieve supra-Sabatier maximum reaction rates and supra-equilibrium conversions and explicated the optimal dynamic catalysis operating conditions for various catalyst types. We conclude this work by considering a short example for finding the optimal catalyst properties to achieve maximum selectivity for an intermediate product for the series reaction shown in Scheme 4. In this reaction, our desired product, B, is an intermediate product en route to $\mathrm{C}$. In this example, the rate constants for adsorption are invariant with $\mathrm{BE}_{\mathrm{B}}$, and $\omega_{j}$ are defined in terms of $\mathrm{BE}_{\mathrm{B}}$ (eq. (29)): 


$$
\omega_{j}=\frac{\Delta \Delta \mathrm{G}_{j}^{\mathrm{o \ddagger}}}{\Delta \mathrm{BE}_{\mathrm{B}}}
$$

There are two constraints on $\omega_{j}$ which require that $\Delta G_{\mathrm{A} \rightarrow \mathrm{B}}^{\mathrm{o}}$ and $\Delta G_{\mathrm{B} \rightarrow \mathrm{C}}^{\mathrm{o}}$ are constant. The ten $\omega_{j}$ are summarized in Scheme 4 with these thermodynamic constraints and with the assumption of barrierless sorption with rate constants that are independent of $\mathrm{BE}_{\mathrm{B}}$. There are four independent $\omega_{j}\left(\omega_{-1}, \omega_{2}, \omega_{4}\right.$, and $\omega_{5}$ ), and the problem of optimizing selectivity towards B initially appears complex. However, by following the same strategies detailed above, the dynamic catalyst properties that maximize selectivity are easily determined.

Scheme 4. Multi-product five-step reaction sequence.

$\begin{array}{ccc}\text { Reaction } & \omega_{j} & \omega_{-j} \\ \text { 1. A }+* \rightleftarrows \mathrm{A}^{*} & 0 & \omega_{-1} \\ \text { 2. } \mathrm{A}^{*} \rightleftarrows \mathrm{B}^{*} & \omega_{2} & 1-\omega_{-1}+\omega_{2} \\ \text { 3. } \mathrm{B}^{*} \rightleftarrows \mathrm{B}+* & 1 & 0 \\ \text { 4. } \mathrm{B}^{*} \rightleftarrows \mathrm{C}^{*} & \omega_{4} & \omega_{5}-1+\omega_{4} \\ \text { 5. } \mathrm{C}^{*} \rightleftarrows \mathrm{C}+* & \omega_{5} & 0\end{array}$

The selectivity towards B at dynamic equilibrium is defined by the dynamic equilibrium constant for the $\mathrm{A} \rightarrow \mathrm{B}$ and the $\mathrm{C} \rightarrow \mathrm{B}$ reactions — both of which need to be large for high selectivity and yield of $\mathrm{B}$. The dynamic equilibrium constants for the reaction A to B is unchanged from the example for Scheme 2 and is thus given by eq. (21) and depends only on $\omega_{-1}, \omega_{2}$, and $\Delta \mathrm{BE}_{\mathrm{B}}$. Likewise, the dynamic equilibrium constant for the $\mathrm{C}$ to $\mathrm{B}$ reaction depends only on $\omega_{4}, \omega_{5}$, and $\Delta \mathrm{BE} \mathrm{E}_{\mathrm{B}}$ (eq. (30)):

$$
\frac{K_{\mathrm{dyn}}^{\mathrm{C} \rightarrow \mathrm{B}}}{K^{\mathrm{C} \rightarrow \mathrm{B}}}=\frac{a_{\mathrm{B}}^{\mathrm{eq}}}{a_{\mathrm{c}}^{\mathrm{eq}}}=\frac{\left\langle k_{-5}\right\rangle\left\langle k_{-4}\right\rangle\left\langle k_{3}\right\rangle}{\left\langle k_{5}\right\rangle\left\langle k_{4}\right\rangle\left\langle k_{-3}\right\rangle}=\frac{\left(1+\exp \left(-\left(\omega_{5}-1+\omega_{4}\right) \frac{\Delta \mathrm{BE}_{\mathrm{B}}}{R T}\right)\right)\left(1+\exp \left(-\frac{\Delta \mathrm{BE}}{R T}\right)\right)}{\left(1+\exp \left(-\omega_{5} \frac{\Delta \mathrm{BE}}{R T}\right)\right)\left(1+\exp \left(-\omega_{4} \frac{\Delta \mathrm{BE}_{\mathrm{B}}}{R T}\right)\right)}
$$

where we consider here a symmetric square-wave oscillation. Since $K_{\mathrm{dyn}}^{\mathrm{C} \rightarrow \mathrm{B}}$ (eq. (30)) and $K_{\mathrm{dyn}}^{\mathrm{A} \rightarrow \mathrm{B}}($ eq. (21)) are independent (besides a mutual dependence on $\Delta \mathrm{BE}_{\mathrm{B}}$ ), there must exist a set of $\omega_{j}$ that gives $100 \%$ 
selectivity and yield of $\mathrm{B}$ at $\Delta \mathrm{BE}_{\mathrm{B}} \rightarrow \infty$. Equation (30) is plotted as a function of $\omega_{4}$ and $\omega_{5}$ in Figure 12, with the iso-equilibrium lines shown as dashed lines and lines that separate regions where $\omega_{j}$ change signs are shown as solid lines. Figure 12 (and eq. (30)) is identical to Figure 1b (and eq. (21)) with the substitutions $\omega_{4}=\omega_{-1}$ and $\omega_{5}=\omega_{2}+1-\omega_{-1}$. Based on the results detailed hereinbefore for maximizing $K_{\mathrm{dyn}}^{\mathrm{A} \rightarrow \mathrm{B}}$ and the results shown in Figure 12 for maximizing $K_{\mathrm{dyn}}^{\mathrm{C} \rightarrow \mathrm{B}}$, a dynamic catalyst with $\omega_{4}<$ $1, \omega_{5}<1$, and $\omega_{5}<1-\omega_{4}$ will give $K_{\mathrm{dyn}}^{\mathrm{C} \rightarrow \mathrm{B}}>K^{\mathrm{C} \rightarrow \mathrm{B}}$, while $\omega_{-1}<1, \omega_{2}<0$, and $\omega_{2}<\omega_{-1}$ gives $K_{\mathrm{dyn}}^{\mathrm{A} \rightarrow \mathrm{B}}>K^{\mathrm{A} \rightarrow \mathrm{B}}$. When all these criteria are satisfied, selectivity and yield of $\mathrm{B}$ are driven to $100 \%$ as $\Delta \mathrm{BE}_{\mathrm{B}} \rightarrow \infty$

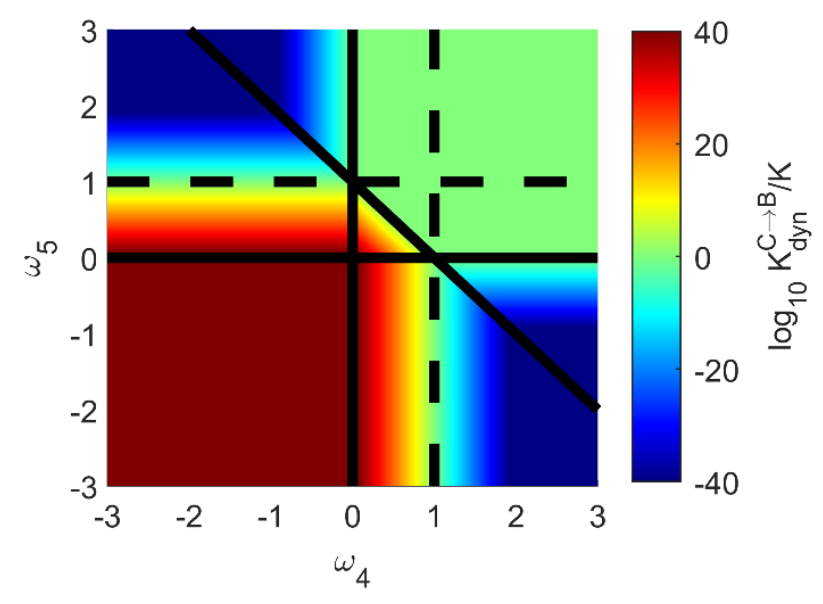

Figure 12. $K_{\mathrm{dyn}}$ for the conversion of $\mathrm{C}$ to $\mathrm{B}$ in Scheme 4 as a function of $\omega_{4}$ and $\omega_{5}$ for a square-wave oscillation with $\Delta \mathrm{BE}_{\mathrm{B}} / R T=100$.

\section{Conclusion}

Dynamic catalysis proffers the ability to obtain greater reaction rates, conversions, and selectivities than static catalysis, but a precise description of the necessary catalyst properties for achieving this has heretofore been unestablished. This work explores dynamic catalysis behavior in the context of linear free energy (LFE) relationships that give rise to traditional Sabatier volcano plot behavior in static catalysis. The ability of a dynamic catalyst to drive conversion beyond the thermodynamic limit, increase the reaction rate, and increase selectivity is demonstrated to be rigorously identifiable in terms of simple governing 
relationships between LFE scaling parameters. In particular, classification of dynamic catalytic properties based on LFE parameters reveals two main classes of dynamic catalysts: (i) those optimized during quasistatic conditions, where the oscillation frequency is sufficiently fast such that the activities of all surface species are approximately constant, and (ii) those optimized during "resonant" conditions, for which enhanced reaction rates and conversions are observed only within a finite resonance frequency band. Within quasi-static dynamic catalysis, we further identify two catalyst sub-types optimized via (i) symmetric or (ii) asymmetric oscillation waveforms. The distinction between symmetric and asymmetric quasi-static catalysts, and the disparate LFE parameters which define their classification, corroborate recent experimental and computational observations that suggest temporal asymmetry can be a powerful lever to enhance rate, conversion, and selectivity in dynamic catalysis.

Derivation of closed-form expressions for rate and conversion enhancement during dynamic catalysis demonstrate that there is an intimate connection between the LFE parameters required to (i) overcome the Sabatier limit to turnover rate and (ii) circumvent thermodynamically-prescribed limits to conversion, quantified by comparison of the dynamic equilibrium constant, $K_{\mathrm{dyn}}$, to the thermodynamic equilibrium constant, $K$. We derive simple, closed-form expressions for $K_{\text {dyn }}$ under quasi-static conditions to demonstrate that $K_{\mathrm{dyn}}$ is an exponential function of the oscillation amplitude. Thus, with the optimal LFE scaling parameters, a dynamic catalyst can theoretically drive any reaction to $100 \%$ conversion, regardless of stringency of limits set by reaction thermodynamics. Insights drawn from analytical expressions of $K_{\text {dyn }}$ extend to product selectivities for which we derive the exact conditions required for $100 \%$ selectivity of any specified intermediate or terminal species.

From detailed study of multiple dynamic catalytic systems, we conclude that the mechanism of resonance in dynamic catalysis is reaction-network specific and may require a case-by-case understanding to develop generalizable theories for identifying which conditions give rise to finite frequency bands with enhanced reaction rates. The necessary occurrence of resonance phenomena at conditions where the thermodynamic activities of species change more slowly than the frequency of oscillation requires 
integration of differential equations to find time-averaged rates of reaction, which may not always have analytical solutions for non-linear reaction schemes. Furthermore, resonance-regime dynamic catalysis have been demonstrated to converge to multiple limit cycles (i.e. multiple dynamic steady states), introducing significant challenges that may arise in the analysis and control of interconnected and/or nonlinear reaction schemes.

\section{Acknowledgments}

This work was performed in part under the auspices of the U.S. Department of Energy by Lawrence Livermore National Laboratory under Contract DE-AC52-07NA27344. 


\section{References}

[1] A.J. Medford, A. Vojvodic, J.S. Hummelshøj, J. Voss, F. Abild-Pedersen, F. Studt, T. Bligaard, A. Nilsson, J.K. Nørskov, From the Sabatier principle to a predictive theory of transition-metal heterogeneous catalysis, J. Catal. 328 (2015) 36-42. doi:10.1016/j.jcat.2014.12.033.

[2] J. Greeley, J.K. Nørskov, M. Mavrikakis, Electronic structure and catalysis on metal surfaces, Annu. Rev. Phys. Chem. 53 (2002) 319-348. doi:10.1146/annurev.physchem.53.100301.131630.

[3] J. Greeley, Theoretical Heterogeneous Catalysis: Scaling Relationships and Computational Catalyst Design, Annu. Rev. Chem. Biomol. Eng. 7 (2016) 605-635. doi:10.1146/annurev-chembioeng080615-034413.

[4] A.K. Vijh, Sabatier-Balandin interpretation of the catalytic decomposition of nitrous oxide on metaloxide semiconductors, J. Catal. 31 (1973) 51-54. doi:10.1016/0021-9517(73)90269-8.

[5] A.A. Balandin, The nature of active centers and the kinetics of catalytic dehydrogenation, Adv. Catal. 10 (1958) 96-129. doi:10.1016/S0360-0564(08)60405-8.

[6] J.L. Lin, I. Wheeldon, Kinetic enhancements in DNA-enzyme nanostructures mimic the sabatier principle, ACS Catal. 3 (2013) 560-564. doi:10.1021/cs300766d.

[7] M.A. Barteau, Linear free energy relationships for $\mathrm{C}_{1}$-oxygenate decomposition on transition metal surfaces, Catal. Letters. 8 (1991) 175-184. doi:10.1007/BF00764114.

[8] D.Y. Kuo, H. Paik, J. Kloppenburg, B. Faeth, K.M. Shen, D.G. Schlom, G. Hautier, J. Suntivich, Measurements of Oxygen Electroadsorption Energies and Oxygen Evolution Reaction on $\mathrm{RuO}_{2}$ (110): A Discussion of the Sabatier Principle and Its Role in Electrocatalysis, J. Am. Chem. Soc. 140 (2018) 17597-17605. doi:10.1021/jacs.8b09657.

[9] R.R. Chianelli, G. Berhault, P. Raybaud, S. Kasztelan, J. Hafner, H. Toulhoat, Periodic trends in 
hydrodesulfurization: In support of the Sabatier principle, Appl. Catal. A Gen. 227 (2002) 83-96. doi:10.1016/S0926-860X(01)00924-3.

[10] J. Kari, J.P. Olsen, K. Jensen, S.F. Badino, K.B.R.M. Krogh, K. Borch, P. Westh, Sabatier Principle for Interfacial (Heterogeneous) Enzyme Catalysis, ACS Catal. 8 (2018) 11966-11972. doi:10.1021/acscatal.8b03547.

[11] B.R. Goldsmith, J. Esterhuizen, J.X. Liu, C.J. Bartel, C. Sutton, Machine learning for heterogeneous catalyst design and discovery, AIChE J. 64 (2018) 2311-2323. doi:10.1002/aic.16198.

[12] M. Andersen, A.J. Medford, J.K. Nørskov, K. Reuter, Analyzing the Case for Bifunctional Catalysis, Angew. Chemie. 128 (2016) 5296-5300. doi:10.1002/ange.201601049.

[13] Y. Li, Q. Sun, Recent Advances in Breaking Scaling Relations for Effective Electrochemical Conversion of $\mathrm{CO}_{2}$, Adv. Energy Mater. 6 (2016) 1-19. doi:10.1002/aenm.201600463.

[14] M.J. Husley, C. Wei Lim, N. Yan, Promoting heterogeneous catalysis beyond catalyst design, Chem. Sci. 11 (2020) 1456-1468. doi:10.1039/c9sc05947d.

[15] M. Shetty, A. Walton, S.R. Gathmann, M.A. Ardagh, J. Gopeesingh, J. Resasco, T. Birol, Q. Zhang, M. Tsapatsis, D.G. Vlachos, P. Christopher, C.D. Frisbie, O.A. Abdelrahman, P.J. Dauenhauer, The Catalytic Mechanics of Dynamic Surfaces: Stimulating Methods for Promoting Catalytic Resonance, ACS Catal. 10 (2020) 12666-12695. doi:10.1021/acscatal.0c03336.

[16] B.L. Foley, N.K. Razdan, Dynamic Catalysis Fundamentals: I. Fast calculation of limit cycles in dynamic catalysis, ChemRxiv. (2021).

[17] G.R. Wittreich, S. Liu, P.J. Dauenhauer, D.G. Vlachos, Catalytic resonance of ammonia synthesis by simulated dynamic ruthenium crystal strain, Sci. Adv. 8 (2022) 1-10. doi:10.1126/sciadv.abl6576. 
[18] M.A. Ardagh, T. Birol, Q. Zhang, O.A. Abdelrahman, P.J. Dauenhauer, Catalytic resonance theory: superVolcanoes, catalytic molecular pumps, and oscillatory steady state, Catal. Sci. Technol. 9 (2019) 5058-5076. doi:10.1039/c9cy01543d.

[19] M.A. Ardagh, M. Shetty, A. Kuznetsov, Q. Zhang, P. Christopher, D.G. Vlachos, O.A. Abdelrahman, P.J. Dauenhauer, Catalytic resonance theory: parallel reaction pathway control, Chem. Sci. 11 (2020) 3501-3510. doi:10.1039/c9sc06140a.

[20] B. Robertson, R.D. Astumian, Frequency dependence of catalyzed reactions in a weak oscillating field, J. Chem. Phys. 94 (1991) 7414-7419. doi:10.1063/1.460173.

[21] R.D. Astumian, B. Robertson, Imposed Oscillations of Kinetic Barriers Can Cause an Enzyme To Drive a Chemical Reaction Away from Equilibrium, J. Am. Chem. Soc. 115 (1993) 11063-11068. doi:10.1021/ja00077a001.

[22] J. Gopeesingh, M.A. Ardagh, M. Shetty, S.T. Burke, P.J. Dauenhauer, O.A. Abdelrahman, Resonance-Promoted Formic Acid Oxidation via Dynamic Electrocatalytic Modulation, ACS Catal. 10 (2020) 9932-9942. doi:10.1021/acscatal.0c02201.

[23] F. Sordello, F. Pellegrino, M. Prozzi, C. Minero, V. Maurino, Controlled Periodic Illumination Enhances Hydrogen Production by over 50\% on Pt/TiO 2 , ACS Catal. 11 (2020) 6484-6488. doi:10.1021/acscatal.1c01734.

[24] C.W. Lim, M.J. Hülsey, N. Yan, Non-Faradaic Promotion of Ethylene Hydrogenation under Oscillating Potentials, JACS Au. 1 (2021) 536-542. doi:10.1021/jacsau.1c00044.

[25] J. Qi, J. Resasco, H. Robatjazi, I.B. Alvarez, O. Abdelrahman, P. Dauenhauer, P. Christopher, Dynamic Control of Elementary Step Energetics via Pulsed Illumination Enhances Photocatalysis on Metal Nanoparticles, ACS Energy Lett. 5 (2020) 3518-3525. doi:10.1021/acsenergylett.0c01978. 
[26] D.S. Liu, R.D. Astumian, T.Y. Tsong, Activation of $\mathrm{Na}^{+}$and $\mathrm{K}^{+}$pumping modes of $(\mathrm{Na}, \mathrm{K})$-ATPase by an oscillating electric field, J. Biol. Chem. 265 (1990) 7260-7267. doi:10.1016/s00219258(19)39108-2.

[27] M.A. Ardagh, O.A. Abdelrahman, P.J. Dauenhauer, Principles of Dynamic Heterogeneous Catalysis: Surface Resonance and Turnover Frequency Response, ACS Catal. 9 (2019) 6929-6937. doi:10.1021/acscatal.9b01606.

[28] R.D. Astumian, C. Pezzato, Y. Feng, Y. Qiu, P.R. Mcgonigal, C. Cheng, J.F. Stoddart, Nonequilibrium kinetics and trajectory thermodynamics of synthetic molecular pumps, Mater. Chem. Front. 4 (2020) 1304-1314. doi:10.1039/d0qm00022a.

[29] G. Ashkenasy, T.M. Hermans, S. Otto, A.F. Taylor, Systems chemistry, Chem. Soc. Rev. 46 (2017) 2543-2554. doi:10.1039/c7cs00117g.

[30] V. Serreli, C.F. Lee, E.R. Kay, D.A. Leigh, A molecular information ratchet, Nature. 445 (2007) 523-527. doi:10.1038/nature05452.

[31] S. Ichikawa, Harmonious optimum conditions for heterogeneous catalytic reactions derived analytically with Polanyi relation and Bronsted relation, J. Catal. 404 (2021) 706-715. doi:10.1016/j.jcat.2021.09.018.

[32] C.T. Campbell, The Degree of Rate Control: A Powerful Tool for Catalysis Research, ACS Catal. 7 (2017) 2770-2779. doi:10.1021/acscatal.7b00115.

[33] S. Kozuch, S. Shaik, A combined kinetic-quantum mechanical model for assessment of catalytic cycles: Application to cross-coupling and Heck reactions, J. Am. Chem. Soc. 128 (2006) 33553365. doi:10.1021/ja0559146.

[34] B.L. Foley, A. Bhan, Degree of rate control and De Donder relations-An interpretation based on 
transition state theory, J. Catal. 384 (2020) 231-251. doi:10.1016/j.jcat.2020.02.008.

[35] S.R. Gathmann, M.A. Ardagh, P.J. Dauenhauer, Catalytic resonance theory: Negative dynamic surfaces for programmable catalysts, Chem Catal. 2 (2022) 140-163. doi:10.1016/j.checat.2021.12.006. 